\title{
Fractionation of lignocellulosic biomass to produce uncondensed aldehyde-stabilized lignin
}

\author{
Masoud Talebi Amiri $\mathbb{1}^{1,2}$, Graham R. Dick $\mathbb{D}^{1,2}$, Ydna M. Questell-Santiago $\mathbb{D}^{1}$ and \\ Jeremy S. Luterbacher $\mathbb{B}^{1{ }^{\prime} \star}$
}

Lignin is one of the most promising sources of renewable aromatic hydrocarbons. Current methods for its extraction from lignocellulosic biomass-which include the kraft, sulfite, and organosolv processes-result in the rapid formation of carbon-carbon bonds, leading to a condensed lignin that cannot be effectively depolymerized into its constituent monomers. Treatment of lignocellulosic biomass with aldehydes during lignin extraction generates an aldehyde-stabilized lignin that is uncondensed and can be converted into its monomers at near-theoretical yields. Here, we outline an efficient, reproducible, and scalable process for extracting and purifying this aldehyde-stabilized lignin as a solid, which can easily be re-dissolved in an organic solvent. Upon exposure to hydrogenolysis conditions, this material provides near-theoretical yields of aromatic monomers ( $40-50 \%$ of the Klason lignin for a typical hardwood). Cellulose and hemicellulose are also efficiently fractionated. This protocol requires 6-7 $\mathrm{h}$ for the extraction of the stabilized lignin and a basic proficiency in synthetic chemistry.

Hydrocarbons are one of the greatest sources of reduced carbon on this planet ${ }^{1}$. Easily extracted, these resources have been exploited for the production of the fuels, chemicals, and materials that underpin our modern societies. However, increasing environmental issues linked to their extraction and use have led us to seek renewable sources of reduced carbon ${ }^{2,3}$. Lignocellulosic biomass is the most abundant form of terrestrial biomass and, as such, is a massive source of renewable reduced carbon $^{4}$. More than $80 \%$ of the mass of this material comes from three of its constituent biopolymers: lignin (15-30\% (wt/wt), dry basis), cellulose (35-55\% (wt/wt), dry basis), and hemicellulose (10-35\% (wt/wt), dry basis $)^{5}$. The monomers of these biopolymers represent potential feedstocks for our future chemical industry and include glucose from cellulose, predominantly xylose from hemicellulose, and aromatic molecules from lignin ${ }^{6}$. Although both glucose and xylose feed into already-developed biorefinery product streams ${ }^{7-9}$, the upgrading of lignin to useful products has not achieved the same success, despite the tremendous need for renewable aromatic molecules ${ }^{10}$. This is largely due to the challenges associated with separating and depolymerizing the polyaromatic biopolymer into its constituent monomers.

\section{Current biomass valorization strategies}

Current biomass deconstruction and valorizing schemes, which mainly include pulp and paper processes and those of emerging biorefineries, generally feature a lignin separation and modification stage, as these processes view lignin as an impediment to the upgrading of the cellulose and hemicellulose fractions ${ }^{11}$. Kraft and sulfite pulping are the dominant technologies in pulp and paper processing. During the kraft process, the biomass is cooked in an aqueous mixture of sodium hydroxide and sodium sulfide; during the Sulfite process, the biomass is typically heated with an aqueous magnesium bisulfite solution at a $\mathrm{pH}$ of either 1.5 or 4.0 (ref. ${ }^{12}$ ). Many biorefinery processes involve treating the raw biomass with mineral acids at high temperature in water ${ }^{13}$, ionic liquids ${ }^{14}$, or various organic solvents ${ }^{15,16}$. Although these strategies are effective at removing lignin, they negatively affect its depolymerization into its constituent monomers post separation ${ }^{17}$. During extraction, the benzylic alcohols of lignin can easily be protonated and eliminated, producing reactive benzylic carbocations that can undergo a subsequent electrophilic aromatic substitution with nearby electron-

${ }^{1}$ Laboratory of Sustainable and Catalytic Processing, Institute of Chemical Sciences and Engineering, École Polytechnique Fédérale de Lausanne (EPFL), Lausanne, Switzerland. ${ }^{2}$ These authors contributed equally: Masoud Talebi Amiri, Graham R. Dick. ${ }^{\star}$ e-mail: jeremy.luterbacher@epfl.ch 


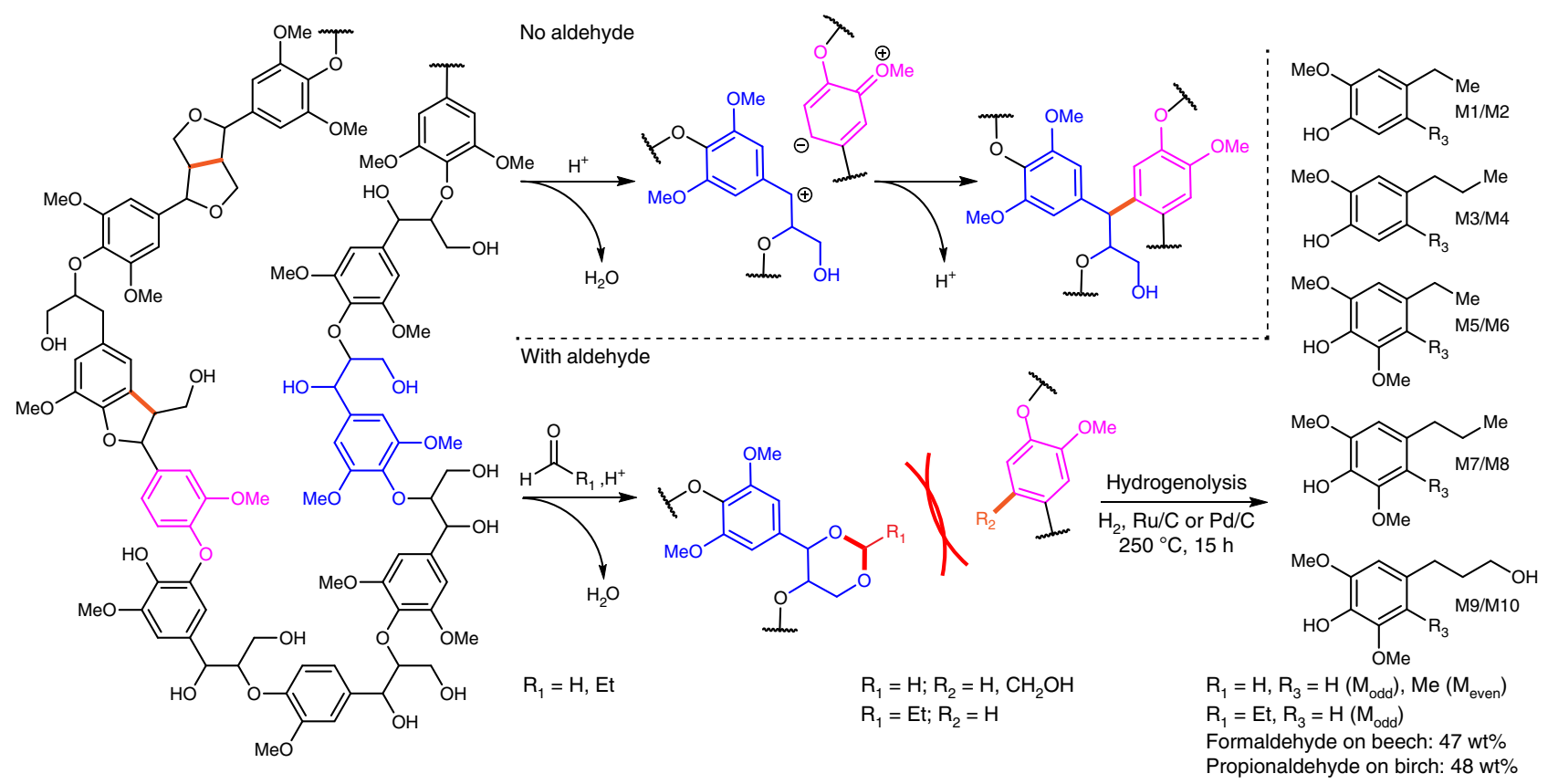

Fig. 1 | Chemical pathways during lignin extraction and valorization. Left, uncondensed lignin biopolymer illustrating the free diol (blue) and the electron-rich guaiacyl subunit (pink). Top, if no aldehyde is used during the extraction, the benzylic alcohol of the free diol is eliminated, producing a benzylic carbocation that undergoes electrophilic aromatic substitution with the guaiacyl or syringyl subunit. The formation of this $\mathrm{C}-\mathrm{C}$ bond is irreversible, preventing the depolymerization of the extracted lignin. Bottom, when an aldehyde is present, the formation of the 1,3-dioxane with the free diol prevents this elimination and thereby the electrophilic aromatic substitution with the guaiacyl or syringyl subunits. Right, the hydrogenolysis of the extracted lignin yields a variety of monomers. If formaldehyde is used, hydroxymethylation of the guaiacyl and syringyl subunits is possible, and ten monomers are typically produced. If propionaldehyde is used, five monomers are generally produced. 4-(3-hydroxypropyl)-2-methoxyphenol and its methylated derivative were not observed as products of the biomass used in these procedures.

rich guaiacyl and syringyl subunits (Fig. 1, top; ref. ${ }^{18}$ ), which leads to the formation of a C-C linkage. Some studies have also depicted the formation of unsaturated guaiacyl or syringyl propene intermediates that similarly condense ${ }^{19}$. Once these $\mathrm{C}-\mathrm{C}$ bonds are formed, their stability leads to low monomer yields after extraction and hydrogenolysis (generally $<5-10 \%$ (wt/wt) of the original Klason lignin content $)^{20,21}$. The separation of lignins from cellulose and hemicellulose is essential before their use in many applications, but is particularly important in pulp and paper processes (in which pure cellulose is required) and before the enzymatic hydrolysis of cellulose (in which lignin can suppress yields of glucose $)^{22}$. Therefore, to valorize lignin, it is essential to develop a fractionation strategy that efficiently separates it from the cellulose and hemicellulose components of biomass while preventing its condensation.

\section{Development of the biomass fractionation procedures}

Recently, we introduced a procedure that facilitates the fractionation of lignocellulosic biomass into its three major components by using formaldehyde as a protecting group for the lignin during its extraction $^{23}$. During this fractionation, the free diol on the lignin side chain (Fig. 1, bottom) is converted into an acetal (1,3-dioxane, shown in red in the figure) by reaction with formaldehyde, thereby preventing the elimination of the benzylic alcohol of that side chain during the acidic extraction. In addition, partial hydroxymethylation occurs on both the electron-rich guaiacyl and syringyl species found in lignin. These transformations prevent the formation of interunit $\mathrm{C}-\mathrm{C}$ bonds by eliminating the electrophilic aromatic substitution pathway. When applied to a sample of beech wood, the resulting extracted lignin provides monomers in a 47\% (wt/wt) yield after hydrogenolysis (on the basis of the original Klason lignin content). To our knowledge, this represented the first instance in which chemically extracted lignin was upgraded at near-theoretical yields on the basis of the original Klason lignin of the lignocellulosic biomass.

More recently, we surveyed a variety of other protecting groups, including aldehydes, ketones, boronic acids, and alkyl carbonates, and discovered that linear aldehydes such as propionaldehyde could similarly facilitate the extraction of uncondensed lignin, achieving a $48 \%$ (wt/wt) yield of 


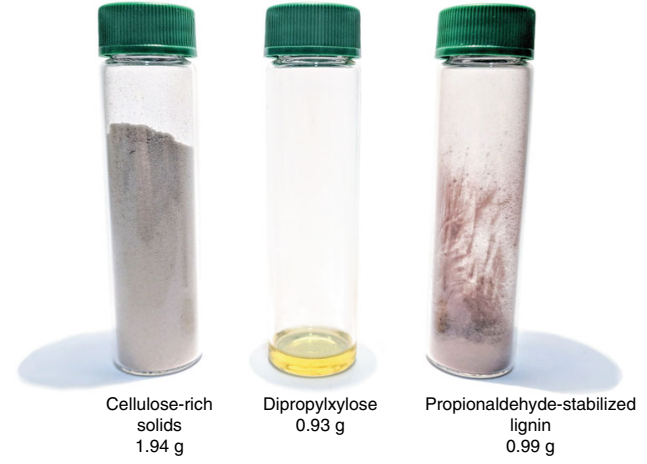

Fig. 2 | The products of the propionaldehyde fractionation procedure (Steps 35-83). The propionaldehyde fractionation procedure was used to fractionate birch wood (extracted and dried, $4.5027 \mathrm{~g}$ ) into highly digestible cellulose-rich solids (left), dipropylxylose (center), and propionaldehyde-stabilized lignin (right). These materials were isolated from a single fractionation.

monomers (on the basis of the original Klason lignin content) after hydrogenolysis when applied to a sample of birch wood ${ }^{24}$. Owing to its structure and reactivity, propionaldehyde forms a 1,3-dioxane structure but does not hydroxyalkylate the aromatic residues. The near-theoretical yield that was obtained despite the absence of hydroxyalkylation indicates that the primary mode of stabilization stems from the suppression of benzylic carbocation formation. This lack of hydroxyalkylation also reduces the diversity of the monomers produced post hydrogenolysis (Fig. 1).

Here, we detail optimized procedures for isolating and purifying both the formaldehyde-stabilized (Steps 1-23) and the propionaldehyde-stabilized (Steps 35-70) lignins in solid form without degrading them by adapting the procedures introduced in the previous publications. Key to the development of these strategies was the determination of the conditions needed to neutralize the reaction solution and the appropriate solvent blends used to facilitate the precipitation and purification of the lignin. These isolated lignins are ideal substrates for further processing or upgrading studies, as they retain their full upgrading potential and can be processed without further effects from the cellulose and hemicellulose fractions. The optimized procedures also describe the recovery of highly digestible cellulose (in the case of the propionaldehyde fractionation, Steps 41-49) and stabilized xylose (Steps 24-34 and Steps 71-83), thereby delineating a methodology to truly fractionate lignocellulosic biomass. Of the biopolymers (as determined by biomass composition analysis ${ }^{25}$ ), $\geq 95 \%$ (wt/wt) were recovered as cellulose-rich solids ( $77-82 \%$ (wt/wt) glucan and $\sim 2-10 \%$ (wt/wt) xylan, representing $87-\geq 95 \mathrm{~mol} \%$ of the native glucan and $6-21 \mathrm{~mol} \%$ of the native xylan), stabilized xylose (solid or liquid, depending on the purity and aldehyde type; $60-78 \mathrm{~mol} \%$ of the native xylan), and solid stabilized lignin (typically representing 103-133\% (wt/wt) of the native Klason lignin, with the extra mass arising from the contribution of acid-soluble lignin and extraneous aldehyde functionalization) (Figs. 2 and 3).

\section{Assessment of lignin valorization strategies}

To evaluate both the extraction and quality of the resulting lignin, we wanted to define a metric to benchmark the total yields of monomers obtained from the extracted and purified lignin for a specific biomass source. In previous work, we have referred to yields of monomers from lignin as a weight percentage of the Klason lignin content of the original biomass, as determined during the sulfuric acid-mediated biomass composition analysis. The monomers' yields are calculated by reconstituting their molecular weight to their pre-hydrodeoxygenated state (e.g., for monomer 1 (M1), the nominal molecular weight is $152.19 \mathrm{~g} \mathrm{~mol}^{-1}$, but it is produced from a subunit of lignin with a molecular weight of $196.20 \mathrm{~g} \mathrm{~mol}^{-1}$; see Equipment setup: 'Monomer yield quantification using gas chromatography'), summing them, and then dividing the sum by the Klason lignin content. By doing so, we, and many others, have reported yields between 40 and 55\% (wt/wt), which is generally assumed to be the theoretical maximum for wild hardwoods ${ }^{22-24,26-29}$. However, as illustrated below (Fig. 4), we have also noted that this measure can vary substantially across biomass sources. In addition, the Klason lignin measurement does not include the acid-soluble lignin fraction, which can be substantial, but is based on an imprecise UV-absorption measurement ${ }^{30}$. This leads to fluctuating yields between species and even more substantially across genera (Fig. 4). 

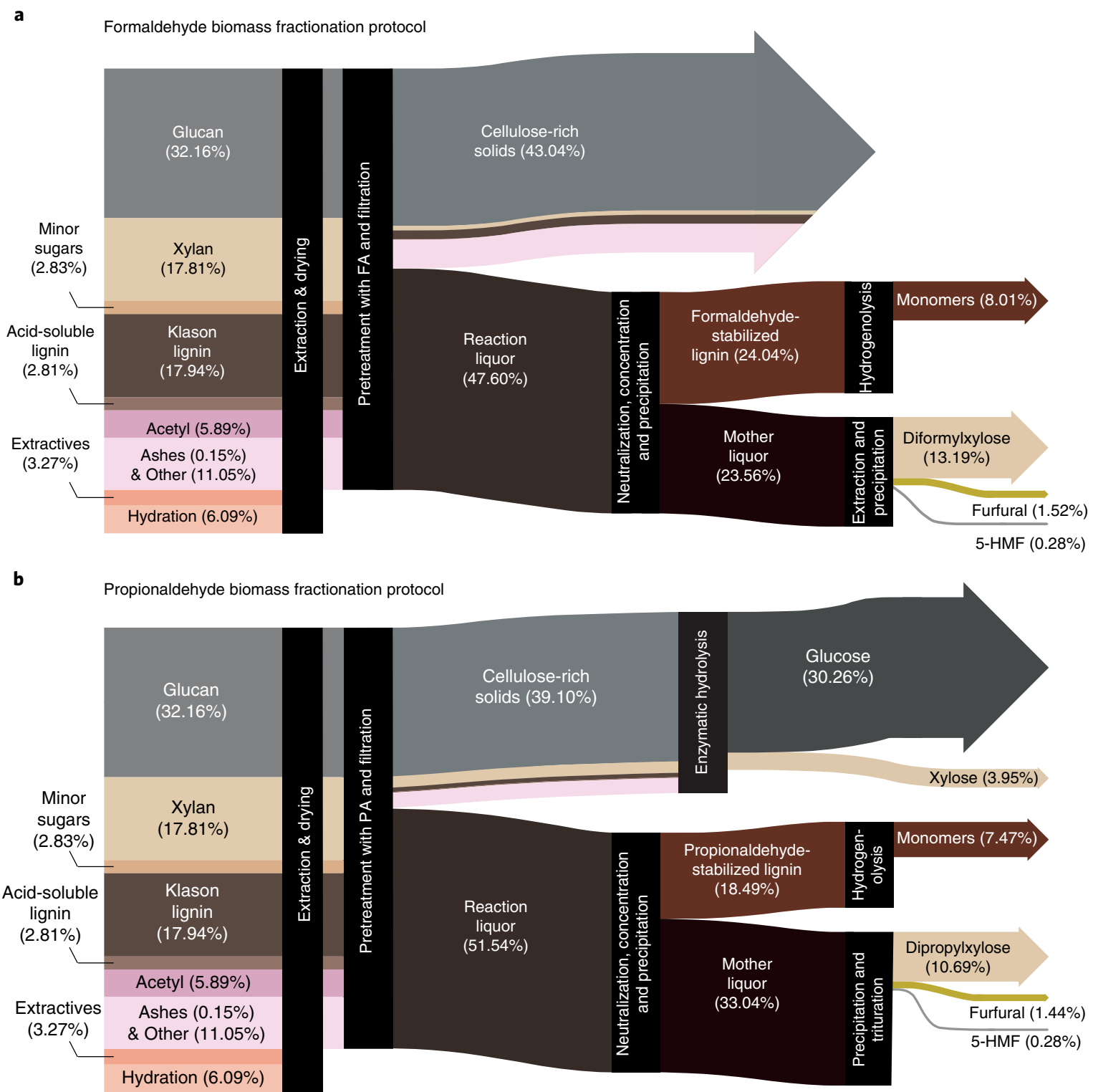

Fig. 3 | Mass balances during the aldehyde fractionation of lignocellulosic biomass, as performed on 2018 birch wood. a,b, The formaldehyde biomass fractionation procedure (a; Steps 1-34) and the propionaldehyde biomass fractionation procedure (b; Steps 35-83). All the numbers are provided as weight per weight percentages. The provided weight per weight percentages of the sugars, stabilized sugars, furfural, hydroxymethylfurfural (HMF), and stabilized lignin have been corrected for the mass of the stabilizing group, as well as hydration, dehydration, or hydrogenation, to match their initial structure in the native biomass. The weight per weight percentages of the monomers have been corrected for the hydrodeoxygenation reactions to reflect their constituent masses as part of the original biopolymer. The initial compositional data represent the average of three samples, and the post pretreatment data come from a single sample. For tabulated forms of these data, see Tables 1-6.

In the past few years, it has been established that the direct hydrogenolysis of the native biomass provides close to the maximum possible yield of obtainable monomers by cleaving the native lignin interunit ether bonds and leaving the interunit $\mathrm{C}-\mathrm{C}$ bonds intact ${ }^{26,29,30}$. Here, we refer to this procedure as 'direct hydrogenolysis', but it is also known as 'reductive fractionation' or 'lignin first $^{19,31,32}$. We argue that this procedure provides the most accurate measure of the theoretical monomer yield from lignin for a given biomass source because it depolymerizes the native lignin's ether linkages before any condensation can occur ${ }^{27,28,33,34}$. We propose that the quality of a given lignin extraction procedure is best determined by comparing the total amount of monomers produced after hydrogenolysis of the extracted lignin to the total amount of monomers produced from the same quantity of native biomass by direct hydrogenolysis. This comparison leads to a rapidly obtained and easily understood metric for determining isolated lignin quality. 
a Dry-biomass weighted

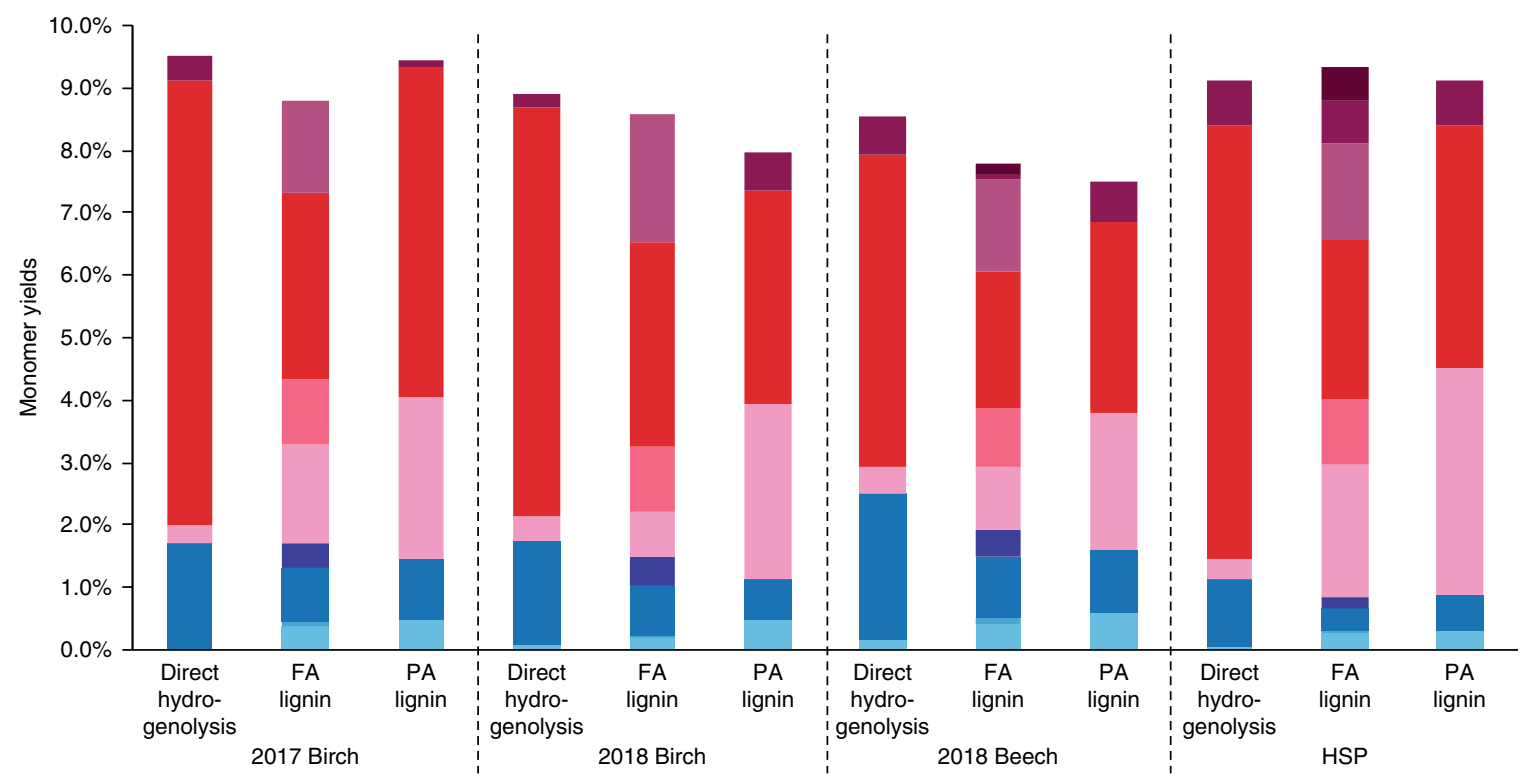

b Klason-lignin weighted
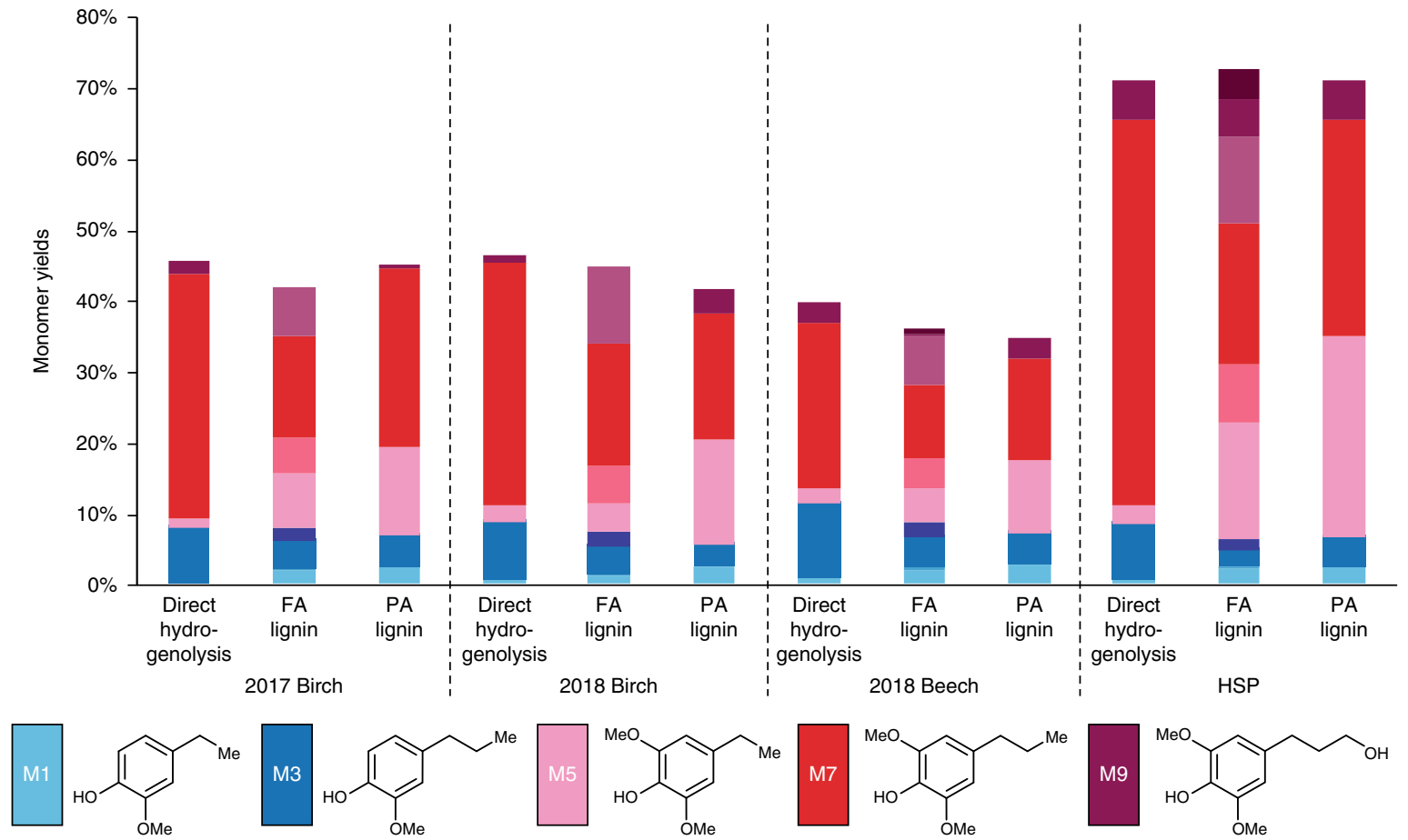<smiles>CCc1cc(OC)c(O)c(OC)c1</smiles>

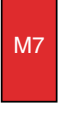<smiles>CCCc1cc(OC)c(O)c(OC)c1</smiles>
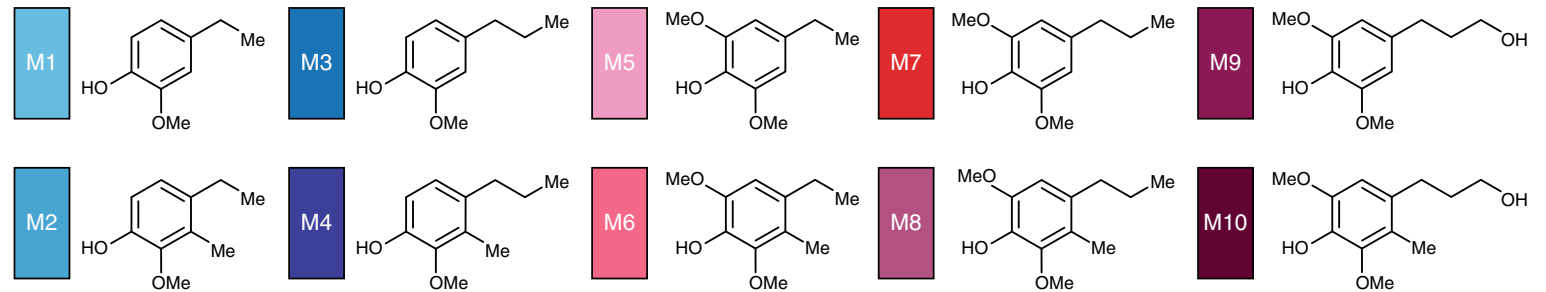

Fig. 4 | Hydrogenolysis data for the formaldehyde and propionaldehyde-stabilized lignins as compared with the direct hydrogenolyses of the feedstock biomass. a,b. These two charts compare the monomer yields from the hydrogenolysis of the raw biomass (direct hydrogenolysis), formaldehyde-stabilized lignin (FA), and propionaldehyde-stabilized lignin (PA) for four biomass sources: 2017 Birch, 2018 Birch, 2018 Beech, and high-syringyl poplar (HSP). The direct hydrogenolysis represents the highest possible yield of monomers for these biomass sources and was performed on biomass that had not been extracted or dried. The formaldehyde and propionaldehyde-stabilized lignins were fractionated from extracted and dried biomass, which typically lowers their yields by 1-2\% (wt/wt) when Klason weighted (b) and $~ 0.25-0.4 \%$ (wt/wt) when wholebiomass weighted (a; Supplementary Fig. 1). Each data point is derived from a single sample. 
Table 1 | Composition of the biomass used for the fractionation procedures

\begin{tabular}{|c|c|c|c|c|c|c|c|c|c|c|c|c|}
\hline $\begin{array}{l}\text { Biomass } \\
\text { type }\end{array}$ & $\mathrm{Ash}^{\mathrm{a}}$ & Hydration $^{a}$ & Extractives $^{a}$ & $\begin{array}{l}\text { Klason } \\
\text { lignin }^{a}\end{array}$ & $\begin{array}{l}\text { Acid-soluble } \\
\text { lignin }^{\text {a }}\end{array}$ & Glucan $^{a}$ & Xylan $^{a}$ & Galactan $^{a}$ & Arabinan $^{a}$ & Mannan $^{a}$ & Acetyl $^{a}$ & Total $^{a}$ \\
\hline Birch $^{b}$ & - & $5.70 \%$ & $2.60 \%$ & $19.60 \%$ & - & - & - & - & - & - & - & - \\
\hline Birch $^{c}$ & $0.15 \%$ & $6.09 \%$ & $3.27 \%$ & $17.94 \%$ & $2.81 \%$ & $32.16 \%$ & $17.81 \%$ & $2.01 \%$ & $0.56 \%$ & $0.26 \%$ & $5.89 \%$ & $88.95 \%$ \\
\hline Beech $^{c}$ & $0.17 \%$ & $6.48 \%$ & $3.06 \%$ & $20.05 \%$ & $2.05 \%$ & $33.20 \%$ & $16.62 \%$ & $1.85 \%$ & $0.50 \%$ & $0.54 \%$ & $7.43 \%$ & $91.95 \%$ \\
\hline $\mathrm{HSP}^{\mathrm{d}}$ & - & $6.4 \%$ & $4.4 \%$ & $12.8 \%$ & $6.4 \%$ & $34.6 \%$ & $14.3 \%$ & - & - & - & - & - \\
\hline
\end{tabular}

Each datum represents the average of three samples. HSP, high-syringyl poplar. ${ }^{a}$ Fractions are presented as a weight percentage of the raw biomass. ${ }^{b}$ Year $2017 .{ }^{c}$ Year $2018 .{ }^{d}$ Data taken from Lan et al. $^{24}$.

Table 2 | Yield of lignin monomers from the direct hydrogenolysis of the biomass on a dry basis

\begin{tabular}{lllllll} 
Biomass type & $\mathbf{M 1}^{\mathbf{a}}$ & $\mathbf{M 3}^{\mathbf{a}}$ & $\mathbf{M 5}^{\mathbf{a}}$ & $\mathbf{M 7}^{\mathbf{a}}$ & $\mathbf{M 9}^{\mathbf{a}}$ & Total $^{\mathbf{a}}$ \\
\hline Birch $^{\mathrm{b}}$ & $0.00 \%$ & $1.69 \%$ & $0.29 \%$ & $7.15 \%$ & $0.39 \%$ & $9.49 \%$ \\
Birch $^{\mathrm{c}}$ & $0.09 \%$ & $1.64 \%$ & $0.42 \%$ & $6.53 \%$ & $0.23 \%$ & $8.91 \%$ \\
Beech $^{\mathrm{c}}$ & $0.16 \%$ & $2.32 \%$ & $0.43 \%$ & $4.99 \%$ & $0.63 \%$ & $8.54 \%$ \\
HSP & $0.05 \%$ & $1.06 \%$ & $0.34 \%$ & $6.96 \%$ & $0.70 \%$ & $9.11 \%$ \\
\hline
\end{tabular}

Each datum is derived from a single sample. HSP, high-syringyl poplar. ${ }^{a}$ Yield is presented as a weight percentage of the total raw biomass on a dry basis and is corrected for any mass lost with respect to the monomers' initial structures in the native lignin polymer. Even-numbered monomers are not seen in the direct hydrogenolysis because there is no hydroxymethylation, as that is only a consequence of the formaldehyde pretreatment. ${ }^{b}$ Year 2017. 'Year 2018.

\section{Advantages and limitations of the approach}

When we subjected the isolated stabilized lignin to hydrogenolysis and, as discussed above, compared the isolated monomer yields to those resulting from the direct hydrogenolysis of native lignin, we observed yields from isolated lignin that are within $\geq 88 \%$ (wt/wt) of those obtained by direct hydrogenolysis without any extraction or fractionation (Fig. 4). This comparison demonstrates that we can isolate and purify a lignin fraction that contains almost all the original native lignin and can be upgraded at near-theoretical yields. In parallel, the isolated cellulose can be enzymatically depolymerized to produce $\geq 94 \mathrm{~mol} \%$ of the glucose when using the propionaldehyde-based stabilization (as compared to the compositional analysis). As previously detailed, cellulose isolated in the presence of formaldehyde has poor digestibility (enzymatic hydrolysis yields $<20 \mathrm{~mol} \%$ ), but can be improved if an acid treatment is performed to remove acetal or formyl species ${ }^{23}$. Furthermore, $\geq 60 \mathrm{~mol} \%$ of the xylose can be recovered as the aldehyde-stabilized derivative. All of these balances are summarized in Fig. 3 and in Tables 1-6 for both fractionation protocols.

The main emphasis of the procedures described in this protocol is to isolate high-quality, uncondensed, bench-stable lignin using aldehyde stabilization. The only alternative for producing standard uncondensed lignin is to use the cellulolytic lignin isolation method, which requires extensive ball milling, enzymatic treatment, and chemical processing of the wood ${ }^{35}$. The timing for all the necessary operations can exceed $5 \mathrm{~d}$ and can be very difficult to scale. By contrast, the procedures presented here are easily scalable to enable the production of gram quantities of bench-stable lignin. They also require only inexpensive chemicals, common laboratory equipment, a rudimentary understanding of synthetic organic chemistry, and 6-7 $\mathrm{h}$ for the isolation of the stabilized lignin (more for the full fractionation procedure).

The main limitations of these protocols stem from their reliance on organic solvents, which are often toxic and/or flammable; formaldehyde and propionaldehyde, which are toxic; and acids, which are corrosive. Consequently, these procedures require a sufficiently ventilated workspace and appropriate protective equipment. However, these precautions and requirements are no different from those of many common chemical reactions or industrial processes. In addition, these procedures have been performed exclusively on hardwood and softwood biomass sources ${ }^{24}$. Given the similarity of lignin structure across several biomass phyla, we believe that these procedures should function well on other lignocellulosic biomass sources, but we have no experimental evidence to support that. 
Table 3 | Yield of lignin monomers from the hydrogenolysis of the isolated formaldehydestabilized lignin powder

\begin{tabular}{|c|c|c|c|c|c|c|c|c|c|c|c|}
\hline Biomass type & $M 1^{a}$ & $M 2^{a}$ & $M 3^{a}$ & $M 4^{a}$ & $M 5^{a}$ & $M 6^{a}$ & $M 7^{a}$ & $M 8^{a}$ & $M 9^{a}$ & $\mathrm{M}^{\mathrm{a}}$ & Total \\
\hline Birch $^{b}$ & $0.37 \%$ & $0.08 \%$ & $0.84 \%$ & $0.41 \%$ & $1.57 \%$ & $1.04 \%$ & $3.00 \%$ & $1.45 \%$ & $0.00 \%$ & $0.00 \%$ & $8.77 \%$ \\
\hline Birch $^{c}$ & $0.18 \%$ & $0.05 \%$ & $0.80 \%$ & $0.44 \%$ & $0.73 \%$ & $1.03 \%$ & $3.29 \%$ & $2.04 \%$ & $0.00 \%$ & $0.00 \%$ & $8.57 \%$ \\
\hline Beech $^{c}$ & $0.42 \%$ & $0.10 \%$ & $0.96 \%$ & $0.43 \%$ & $1.01 \%$ & $0.93 \%$ & $2.21 \%$ & $1.48 \%$ & $0.07 \%$ & $0.16 \%$ & $7.78 \%$ \\
\hline HSP & $0.25 \%$ & $0.04 \%$ & $0.35 \%$ & $0.21 \%$ & $2.09 \%$ & $1.05 \%$ & $2.56 \%$ & $1.56 \%$ & $0.67 \%$ & $0.54 \%$ & $9.32 \%$ \\
\hline
\end{tabular}

Each datum is derived from a single sample. HSP, high-syringyl poplar. ${ }^{a}$ Yield is presented as a weight percentage of the total raw biomass on a dry basis and is corrected for any mass lost with respect to the monomers' initial structures in the native lignin polymer due to the hydrogenolysis. byear 2017. ' Year 2018.

Table 4 | Yield of lignin monomers from the hydrogenolysis of the isolated propionaldehydestabilized lignin powder

\begin{tabular}{lllllll} 
Biomass type & M1 & M3 & M5 $^{\mathbf{a}}$ & M7 $^{\mathbf{a}}$ & M9 $^{\mathbf{a}}$ & Total $^{\mathbf{a}}$ \\
\hline Birch $^{\mathrm{b}}$ & $0.49 \%$ & $0.98 \%$ & $2.59 \%$ & $5.26 \%$ & $0.11 \%$ & $9.43 \%$ \\
Birch $^{\mathrm{C}}$ & $0.47 \%$ & $0.65 \%$ & $2.83 \%$ & $3.39 \%$ & $0.63 \%$ & $7.97 \%$ \\
Beech $^{c}$ & $0.59 \%$ & $1.00 \%$ & $2.21 \%$ & $3.04 \%$ & $0.64 \%$ & $7.49 \%$ \\
HSP & $0.29 \%$ & $0.58 \%$ & $3.64 \%$ & $3.88 \%$ & $0.71 \%$ & $9.11 \%$ \\
\hline
\end{tabular}

Each datum is derived from a single sample. HSP, high-syringyl poplar. ${ }^{2}$ Yield is presented as a weight percentage of the total raw biomass on a dry basis and is corrected for any mass lost with respect to the monomers' initial structures in the native lignin polymer due to the hydrogenolysis. 'YYear 2017. ' Year 2018

Table 5 | Composition and enzymatic hydrolysis of the extracted cellulose from the aldehyde biomass fractionation procedures

\begin{tabular}{|c|c|c|c|c|c|c|c|c|}
\hline \multirow[b]{2}{*}{$\begin{array}{l}\text { Biomass } \\
\text { type }\end{array}$} & \multirow[b]{2}{*}{$\begin{array}{l}\text { Fractionation } \\
\text { procedure }\end{array}$} & \multicolumn{5}{|c|}{ Compositional analysis } & \multicolumn{2}{|c|}{$\begin{array}{l}\text { Enzymatic } \\
\text { hydrolysis }\end{array}$} \\
\hline & & Glucan & Xylan & Hydration & $\begin{array}{l}\text { Klason } \\
\text { lignin }\end{array}$ & $\begin{array}{l}\text { Acid-soluble } \\
\text { lignin }\end{array}$ & Glucan $^{a}$ & Xylan $^{a}$ \\
\hline Birch $^{b}$ & Formaldehyde & $77.4 \%$ & $2.2 \%$ & $1.7 \%$ & $4.4 \%$ & $0.2 \%$ & $33.8 \%$ & $3.6 \%$ \\
\hline Beech $^{b}$ & Formaldehyde & $79.4 \%$ & $2.6 \%$ & $2.5 \%$ & $3.7 \%$ & $0.3 \%$ & $40.5 \%$ & $4.1 \%$ \\
\hline Birch $^{b}$ & Propionaldehyde & $78.1 \%$ & $6.0 \%$ & $5.2 \%$ & $2.3 \%$ & $0.5 \%$ & $77.4 \%$ & $10.1 \%$ \\
\hline Beech $^{b}$ & Propionaldehyde & $80.6 \%$ & $5.6 \%$ & $3.1 \%$ & $2.0 \%$ & $0.4 \%$ & $82.1 \%$ & $9.3 \%$ \\
\hline
\end{tabular}

The yields in this table are all weight percentages and are presented as the reconstituted versions of their original biopolymers from the monomers that were observed by HPLC (e.g., xylose to xylan, glucose to glucan). Each compositional analysis datum represents the average of two samples, and each

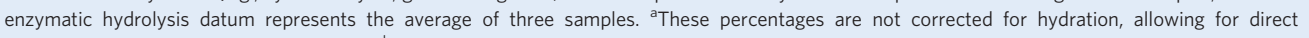
comparison to the compositional analyses. ${ }^{b}$ Year 2018.

\section{Overview of the procedure}

In this protocol, we have provided detailed descriptions of the formaldehyde and propionaldehyde procedures that lead to the full fractionation of lignocellulosic biomass into its three constituent biopolymers as distinct, readily upgradable fractions. To aid in their comprehension, we provide a brief summary of these procedures here. First, the extraction is performed, during which the biomass is heated with the stabilizing aldehyde and hydrochloric acid in 1,4-dioxane at elevated temperatures for 3-3.5 h (Steps 1-4 and Steps 35-40). Once complete, the cellulose-rich solids are collected by filtration and washed with 1,4-dioxane and methanol to remove any residual lignin or stabilized sugars (Steps 5 and 6 and Steps 41 and 42). The filtrate is then set aside, and the cellulose-rich solids 
Table 6 | Masses and yields of isolated fractions from the fractionation procedures

\begin{tabular}{|c|c|c|c|c|c|c|}
\hline Biomass type & $\begin{array}{l}\text { Fractionation } \\
\text { procedure }\end{array}$ & $\begin{array}{l}\text { Cellulose-rich } \\
\text { solids }\end{array}$ & $\begin{array}{l}\text { Protected } C_{5} \\
\text { sugars }^{a}\end{array}$ & $\begin{array}{l}\text { 5-Hydroxymethyl } \\
\text { furfural }\end{array}$ & 2-Furfural & Stabilized lignin \\
\hline Birch $^{b}$ & Formaldehyde & $2.1373 \mathrm{~g}(43.0 \%)$ & $0.8631 \mathrm{~g}(13.2 \%)$ & $0.0101 \mathrm{~g}(0.3 \%)$ & $0.0549 \mathrm{~g}(1.5 \%)$ & $1.2150 \mathrm{~g}(24.0 \%)$ \\
\hline Beech $^{b}$ & Formaldehyde & 1.7548 g (35.3\%) & 0.8517 g (13.0\%) & 0.0137 g (0.4\%) & $0.0665 \mathrm{~g}(1.8 \%)$ & $1.0846 \mathrm{~g}(21.4 \%)$ \\
\hline Birch $^{b}$ & Propionaldehyde & 1.9425 g (39.1\%) & 0.9257 g $(10.7 \%)$ & $0.0083 \mathrm{~g}(0.2 \%)$ & $0.0521 \mathrm{~g}(1.4 \%)$ & $0.9853 \mathrm{~g}(18.5 \%)$ \\
\hline Beech $^{b}$ & Propionaldehyde & $1.8998 \mathrm{~g}(37.9 \%)$ & 0.9011 g (10.3\%) & 0.0122 g $(0.3 \%)$ & $0.0616 \mathrm{~g}(1.7 \%)$ & $1.0976 \mathrm{~g}(20.6 \%)$ \\
\hline
\end{tabular}

Each datum is derived from a single sample. The percentages in this table are all weight percentages and are presented as the reconstituted aldehyde-free versions of their original biopolymers (e.g., xylose to xylan, 5-hydroxymethylfurfural to glucan). ${ }^{a}$ Diformylxylose from the formaldehyde fractionation protocol and dipropylxylose from the propionaldehyde fractionation protocol. ${ }^{\text {b }}$ ear 2018.

are treated with either dilute sulfuric acid, in the case that formaldehyde was used as the stabilizing aldehyde (Steps 7-9), or saturated sodium bicarbonate solution, in the case that propionaldehyde was used as the stabilizing aldehyde (Steps 43-45) to cleave any residual acetals on the substrate. This material is then washed with deionized water and acetone, and then dried in vacuo to yield the purified cellulose (Steps 10-14 and Steps 46-49).

The filtrate that was set aside previously is then neutralized through the addition of either a saturated sodium bicarbonate solution, in the case of formaldehyde (Steps 15-19), or solid sodium bicarbonate, in the case of propionaldehyde (Steps 50 and 51). At this point, these procedures diverge substantially. To recover the formaldehyde-stabilized lignin, the dioxane of the neutralized filtrate is first removed by evaporation (Step 20). This results in the precipitation of the lignin, which is then collected by filtration, washed with deionized water, and dried (Steps 21-23). To recover the formaldehyde-stabilized xylose, the filtrate is extracted with ethyl acetate, and the ethyl acetate fraction is concentrated in vacuo to form a yellow oil. This oil is then added dropwise to a stirred solution of hexanes, resulting in the precipitation of a solid. This solution is then filtered to remove insoluble impurities and concentrated in vacuo to afford the sugar as a yellow oil (Steps 24-34). To recover the propionaldehyde-stabilized lignin, the neutralized filtrate is filtered to remove the bicarbonate, and the filtrate is concentrated in vacuo to form a brown oil (Steps 52-57). This oil is then diluted with ethyl acetate and added dropwise to a stirred solution of hexanes, resulting in the precipitation of a solid (Steps 58 and 59). This solid is collected by filtration and triturated with diethyl ether to afford the propionaldehyde-stabilized lignin (Steps 60-70). To recover the propionaldehyde-stabilized xylose, the hexanes filtrate and diethyl ether supernatant are combined, concentrated in vacuo, and purified by chromatography to afford the sugar as a yellow oil (Steps 71-83).

To determine the quality of the cellulose-rich solids, enzymatic hydrolyses are performed on the purified materials in a $\mathrm{pH} 5$ citrate buffer and compared to their sulfuric acid-mediated compositional analyses (Steps 84-116). The quality and purity of the extracted lignins are determined through both ${ }^{1} \mathrm{H}-\mathrm{NMR}$ and hydrogenolysis (Steps $117-128$ ). The purity of the stabilized xyloses is determined by ${ }^{1} \mathrm{H}-\mathrm{NMR}$.

\section{Experimental design}

Here, we detail optimized procedures for isolating and purifying both the formaldehyde-stabilized and propionaldehyde-stabilized lignins in solid form. Beyond extracting isolated lignins that retain their full upgrading potential, the procedures also allow for the full fractionation of the lignocellulosic biomass, producing highly digestible cellulose (in the case of the propionaldehyde fractionation) and stabilized xylose. Before embarking on these procedures, there are a few considerations that should be made for potential adjustments.

\section{Before the fractionation}

As the biomass source largely dictates the results obtained for the fractionation procedures, it is important to fully characterize the feedstock composition beforehand. With that in mind, we have also detailed methods to quantify the ash, hydration, extractives, structural sugar, and lignin of the biomass. These procedures are based on available protocols and are re-described here in Boxes 1-5 for convenience ${ }^{25,36,37}$. Should certain characteristics of the extracted materials be desired, we 


\section{Box 1 | Biomass ash quantification}

Additional equipment

- Aluminum foil (Fisher Scientific, cat. no. 01-213-101)

- Muffle furnace $\left(100-1,200{ }^{\circ} \mathrm{C}, 220-240 \mathrm{~V}, 1,560 \mathrm{~W}, 50 / 60 \mathrm{~Hz}\right.$; Thermo Scientific, cat. no. F48020-33-80)

- Porcelain crucibles (Haldenwanger, cat. no. $79 \mathrm{MF} / 7$ )

\section{Procedure}

1 Heat three clean, dry crucibles to $120{ }^{\circ} \mathrm{C}$ for $16 \mathrm{~h}$ in an oven

2 Let the crucibles cool in a vacuum desiccator ( $25 \mathrm{mbar}$ ) for $1 \mathrm{~h}$ at room temperature.

3 Tare the crucibles and then mass $1 \mathrm{~g}$ of the biomass into each of the crucibles.

4 Record the new mass of the crucibles and cover each of them with a small square of aluminum foil with holes punched into it.

5 Place the crucibles into a ventilated muffle furnace under air and heat it to $600{ }^{\circ} \mathrm{C}$.

6 Leave the samples for $24 \mathrm{~h}$.

$\triangle$ CRITICAL STEP Any pencil or pen markings will burn off, so note the position of the crucibles on a sheet of paper so that they can be identified upon removal from the muffle furnace.

7 Remove the crucibles and cool them to room temperature in a vacuum desiccator ( $25 \mathrm{mbar})$ for $1 \mathrm{~h}$.

8 Re-mass the crucibles with ashes. Use Eq. (6) to calculate the weight percentage of ashes in the sample.

$$
\operatorname{Ashes}(\%(\mathrm{wt} / \mathrm{wt}))=\left(\frac{m_{\text {Crucible \& ashes }}-m_{\text {Crucible }}}{m_{\text {Crucible \& biomass }}-m_{\text {Crucible }}}\right) \times 100 \%
$$

\section{Box 2 | Biomass hydration quantification}

\section{Procedure}

1 Mass $2 \mathrm{~g}$ of biomass into each of three tared, $50-\mathrm{mL}$ centrifuge tubes.

2 Record the new mass of the centrifuge tubes.

3 Loosely cap and place the tubes into a vacuum oven at $60{ }^{\circ} \mathrm{C}$ and dry them for at least $16 \mathrm{~h}$ in vacuo ( 50 mbar).

4 Remove the biomass from the vacuum oven and cool it in a vacuum desiccator ( $25 \mathrm{mbar}$ ) for $1 \mathrm{~h}$ at room temperature.

5 Re-mass the biomass and calculate the mass loss using Eq. (7). This quantity is the hydration of the biomass. Abbreviations: $m$ mass; CFT, centrifuge tube.

$$
\operatorname{Hydration}(\%(\mathrm{wt} / \mathrm{wt}))=\left(\frac{m_{\text {CFT \& Dry biomass }}-m_{\text {CFT }}}{m_{\text {CFT \& Biomass }}-m_{\text {CFT }}}\right) \times 100 \%
$$

recommend that readers first optimize their choice of biomass using one or more of the available characterization protocols. Of particular concern is the quality of the lignin in the raw biomass (e.g., fraction of native interunit ether linkages versus native interunit $\mathrm{C}-\mathrm{C}$ linkages) ${ }^{30,38}$. If the biomass has been processed in any way (e.g., heated or dried), the lignin that is extracted may already be cross-linked and will therefore provide low yields of monomers upon hydrogenolysis. We highly recommend performing a direct hydrogenolysis (Box 5, Determination of the theoretical monomer yields from biomass) on a sample of the biomass that you intend to extract. If it gives you poor yields, then although the extraction will afford you a stabilized lignin, it will similarly have poor yields when upgraded.

\section{Fractionation}

Here, we present the procedures that are most optimal for hardwood biomass sources. However, depending on the biomass source, these can be modified for markedly improved results. Variables that should be considered include the acid loading, temperature, and duration of the extraction (Steps 2 and 3 or 36-39). We have found that the reaction can tolerate acid ranges of 0.3-1.4 M, temperatures between 75 and $100{ }^{\circ} \mathrm{C}$, and durations of $3-5 \mathrm{~h}$. Modifications outside of those parameters may be necessary, but we have found them to consistently provide optimal results.

In cases of unusual lignin structures, including those that have a lower degree of polymerization and/or a high acid-soluble lignin content, modifications will need to be made to obtain the most optimal results. Given the nature of the formaldehyde-extraction procedure, no modifications will need to be made; however, the propionaldehyde-based procedure may need to be modified to obtain optimal yields. For some biomass sources, the solubility of the extracted propionaldehyde-stabilized 


\section{Box 3 | Biomass extractives quantification}

Additional equipment

- Centrifuge (ventilated benchtop centrifuge; Mega Star 1.6; VWR, cat. no. 521-1749)

\section{Procedure}

1 Mass $2 \mathrm{~g}$ of biomass into each of three tared, $50-\mathrm{mL}$ centrifuge tubes.

2 Record the new mass of the centrifuge tubes.

3 Prepare $400 \mathrm{~mL}$ of $80 \%$ ethanol by mixing $320 \mathrm{~mL}$ of absolute (100\%) ethanol with $80 \mathrm{~mL}$ of Milli-Q water.

4 Add $40 \mathrm{~mL}$ of the $80 \%$ ethanol to each centrifuge tube.

5 Cap the centrifuge tubes and sonicate them at room temperature for $30 \mathrm{~min}$.

6 Centrifuge the tubes for $5 \mathrm{~min}$ at 4,500 g at room temperature to separate the solids from the solution.

7 Decant the solution.

8 Repeat steps 4-7 twice more with $80 \%$ ethanol, three times with Milli-Q water, and once with absolute ethanol.

9 Loosely cap the centrifuge tubes and place the biomass into a vacuum oven at $60{ }^{\circ} \mathrm{C}$; dry it for at least $16 \mathrm{~h}$ in vacuo ( 50 mbar final pressure).

10 Remove the biomass from the vacuum oven and cool it in a vacuum desiccator ( $25 \mathrm{mbar})$ for $1 \mathrm{~h}$ at room temperature.

11 Re-mass the biomass and calculate the mass loss. This quantity includes both the hydration and extractives of the biomass. Calculating the difference between the two mass losses yields the mass of the extractives. See Eq. (8) below to calculate this value. Abbreviations: $m$, mass; CFT, centrifuge tube.

$$
\operatorname{Extractives}(\%(\mathrm{wt} / \mathrm{wt}))=\left(\frac{m_{\text {CFT \& Extracted biomass }}-m_{\text {CFT }}}{m_{\text {CFT \& Biomass }}-m_{\text {CFT }}}\right) \times 100 \%-\text { Hydration }(\%(\mathrm{wt} / \mathrm{wt}))
$$

lignin can be substantially altered. Normally, during the procedure, we perform a final ether trituration of the lignin to extract residual stabilized sugars (Steps 64-70), but unusual lignins may be partially soluble in ether, and performing this step may remove a portion of the lignin, markedly reducing the yield of monomers after subsequent hydrogenolysis. The impact of this phenomenon can be seen during the extraction of lignin from high-syringyl poplar (HSP), in which the low Klason lignin content gives comparatively high hydrogenolysis yields versus other biomass sources when compared on the basis of that component (Fig. 4). This comparative advantage is reduced when the yields are instead compared on the basis of the total biomass. Although this is partially due to the reduced total lignin content in the plant, the higher fraction of acid-soluble lignin content in HSP relative to other biomass sources contributes substantially to this advantage (Table 1). Because of the modified solubility of this unusual lignin, the ether trituration step had to be eliminated for HSP to achieve the high yields presented in Fig. 4 for the extracted propionaldehyde lignin. Depending on your needs, you may wish to avoid this step as well.

\section{Depolymerization of the extracted biopolymers}

Once the biomass is fractionated, the cellulose and lignin can be depolymerized using enzymatic hydrolysis (Steps 84-95) and hydrogenolysis (Steps 117-128), respectively. The cellulose produced from the propionaldehyde-based fractionation and washed with a saturated sodium bicarbonate solution can be used directly for enzymatic hydrolysis, leading to near-quantitative yields of glucose. For formaldehyde-based fractionation, the formaldehyde grafting can markedly impact the enzymatic hydrolysis. Dilute sulfuric acid can cleave the acetals on the cellulose backbone, improving digestibility. Depending on the source biomass and any additional modifications to the procedure, the concentration of the sulfuric acid may need to be varied, along with the temperature and duration of the reaction to obtain the best enzymatic hydrolysis results (Steps 7-9). As for the hydrogenolysis (Steps 117-128), the solvent, temperature, duration, and catalyst loading can markedly impact the yield and distribution of the monomers from the reaction. By contrast, the reaction seems to be insensitive to hydrogen pressure, as we have observed nearly identical monomer yields with pressures as low as 3 bar. However, given that catalyst reducibility can be highly dependent on various factors (e.g., storage conditions, identity of the metal precursors used for the catalyst preparation, time elapsed since preparation), we recommend operating the hydrogenolysis at 40 bar of hydrogen to avoid any issues associated with this variable. Here, we present one set of conditions that should provide a good gauge of the quantity of monomers that can be produced from a given stabilized lignin sample and use this to evaluate the quality of the stabilized lignin. 


\section{Box 4 | Structural sugar (cellulose and hemicellulose), acid-soluble lignin, and Klason lignin quantification}

\section{Additional equipment}

- LC system. Agilent Technologies 1260 Infinity system with 1260 high-performance degasser (Agilent Technologies, model no. G4225A), 1260 binary pump (Agilent Technologies, model no. G1312B), 1260 ALS (Agilent Technologies, model no. G1329B), 1260 TCC (Agilent Technologies, model no. G1316A), 1260 DAD (Agilent Technologies, model no. G4212B), and 1260 RID (Agilent Technologies, model no. G1362A) equipped with an Aminex HPX-87P column (300 mm $\times 7.8$ mm; Bio-Rad, cat. no. 125-0098) and Micro-Guard de-ashing guard column (Bio-Rad, cat. no. 125-0118)

- Bulb pipette (100 mL; Poulten \& Graf, cat. no. 12305104)

- Centrifuge tubes (15 mL; Sarstedt, cat. no. 62.554.502)

- Planetary ball mill (PM 100; Retsch, cat. no. 205400001)

- Reagent bottle with GL 45 polypropylene cap (250 mL; Simax, cat. no. 1632414321250)

\section{Procedure}

1 Prepare $50 \mathrm{~mL}$ of $72 \%(\mathrm{wt} / \mathrm{wt}) \mathrm{H}_{2} \mathrm{SO}_{4}$ (specific gravity $=1.634 \mathrm{~g} \mathrm{~mL}^{-1}$ ) by adding $61.94 \mathrm{~g}$ of concentrated sulfuric acid to $16 \mathrm{~g}$ of deionized water in a $50-\mathrm{mL}$ volumetric flask and then diluting with deionized water to a final solution volume of $50 \mathrm{~mL}$.

! CAUTION This dilution is extremely exothermic. Always add acid to water and not vice versa. Let the solution cool to room temperature before diluting to $50 \mathrm{~mL}$

2 Extract and dry $5 \mathrm{~g}$ of biomass (see Reagent setup: Bulk biomass extractives removal and drying).

3 Ball-mill the biomass for $2 \mathrm{~h}$ at 450 r.p.m., using a $50 \%$ duty cycle ( $5 \mathrm{~min}$ on, $5 \mathrm{~min}$ off) until the biomass is a fine powder.

4 Mass $0.3 \mathrm{~g}$ of the ball-milled biomass into each of three tared, $50-\mathrm{mL}$ centrifuge tubes. These will be used to determine the hydration of the ball-milled biomass. Record the mass of the centrifuge tubes.

5 Add a $0.2-\mu \mathrm{m}$ nylon membrane filter to each of three separate, $50-\mathrm{mL}$, self-standing centrifuge tubes.

6 Place the centrifuge tubes from steps 4 and 5, loosely capped, into a vacuum oven at $60{ }^{\circ} \mathrm{C}$.

7 Mass $0.5 \mathrm{~g}$ of the ball-milled biomass into each of another three tared, 15 -mL centrifuge tubes with oval stir bars $(20-\mathrm{mm}$ long $\times 10-\mathrm{mm}$ diameter) and record the mass of the biomass.

8 Into each centrifuge tube, add $7.5 \mathrm{~mL}$ of $72 \%$ (wt/wt) $(12 \mathrm{M}) \mathrm{H}_{2} \mathrm{SO}_{4}$ using a 1-10-mL variable-volume, single-channel pipette.

9 Cap the centrifuge tubes, shake and vortex them to distribute the solid, and sonicate them for $2 \mathrm{~h}$ at $30^{\circ} \mathrm{C}$.

10 Transfer the contents of the centrifuge tubes quantitatively to $500-\mathrm{mL}$ reagent bottles with $\mathrm{GL} 45$ polypropylene caps using Milli-Q water and dilute the solutions to $\sim 300 \mathrm{~mL}$ with Milli-Q water.

11 Autoclave the bottles for $1 \mathrm{~h}$ at $120^{\circ} \mathrm{C}$.

12 Transfer the hot solutions $\left(\sim 85^{\circ} \mathrm{C}\right)$ to a refrigerator and let them cool overnight.

13 The next day, remove the centrifuge tubes from steps 4 and 5 from the vacuum oven and cool them in a vacuum desiccator ( $25 \mathrm{mbar}$ ) for $1 \mathrm{~h}$ at room temperature.

14 Mass the centrifuge tubes and record the masses. Calculate the hydration of the biomass using the data from step 4 and Eq. (7) from Box 2.

15 Remove the reagent bottles from the refrigerator and filter the solutions through the dried, tared, $0.2-\mu m$ nylon membrane filters from step 5 , washing with Milli-Q water.

16 Place the nylon membrane filters and filter cakes into their corresponding centrifuge tubes and loosely cap those centrifuge tubes. Place them in a vacuum oven at $60{ }^{\circ} \mathrm{C}$ and dry them for $24 \mathrm{~h}$ in vacuo ( $\sim 50$ mbar final pressure).

17 Transfer the filtrates to separate $500-\mathrm{mL}$ volumetric flasks, diluting with Milli-Q water, and then return the filtrates to the 500 - $\mathrm{mL}$ reagent bottles.

18 Mass $\mathrm{NaHCO}_{3}(3 \mathrm{~g}, 35.7 \mathrm{mmol}$ ) into each of three $250-\mathrm{mL}$ reagent bottles.

19 Using a $100-\mathrm{mL}$ Mohr pipette, transfer $100 \mathrm{~mL}$ of each of the diluted acidic filtrates to the reagent bottles with $\mathrm{NaHCO}_{3}$.

20 Once neutralized, for each sample, remove an aliquot from the neutralized filtrate and filter it through a syringe filter into an HPLC autosampler vial and cap it. Also, for each sample, filter an aliquot of the acidic filtrate into an HPLC autosampler vial and cap it. Analyze the samples by HPLC, using the pH 7 and $\mathrm{pH} 2$ methods described in the Equipment setup to determine the concentration of $\mathrm{D}-(+)$ glucose, $\mathrm{D}-(+)$ xylose, $\mathrm{D}-(+)$ galactose, $\mathrm{L}-(+)$ arabinose, and $\mathrm{D}-(+)$ mannose, 2-furfural, acetic acid, and 5-hydroxymethylfurfural in the filtrate. When presenting the data, add the HPLC responses (grams per liter) of 5-hydroxymethylfurfural and 2-furfural reconstituted as glucose (multiply the 5-hydroxymethylfurfural signal by 1.429) and xylose (multiply the 2 -furfural signal by 1.563) to the observed yields for those of glucose and xylose. Use the following generalized Eq. (9) to calculate the contribution of each sugar to the overall mass of the material. To determine the acetyl content, multiply the HPLC responses for acetic acid by 0.7169. Abbreviations: m, mass; HPLC, high-pressure liquid chromatography; MW, molecular weight; BMB, ball-milled biomass; RBM, raw biomass; $H$, hydration; $E$, extractives.

$$
\text { Sugar polymer }(\%(\mathrm{wt} / \mathrm{wt}))=\left(\frac{\left[H P L C \text { response }\left(g \cdot \mathrm{L}^{-1}\right)\right] \times(1 L) \times\left(\frac{M w_{\text {sugar }}-M W_{\text {vater }}}{M \text { sugar }}\right)}{m_{B M B} \times\left(1-H_{B M B}(\%(w t / w t))\right.}\right) \times\left(1-H_{R B M}(\%(w t / w t))-E_{R B M}(\%(w t / w t))\right) \times 100 \%
$$

21 Obtain a UV absorbance trace of the acidic diluted filtrate from 190 to $300 \mathrm{~nm}$, using a quartz cuvette. Record the absorbance at $205 \mathrm{~nm}$. If the absorbance exceeds 2, dilute the solution with $0.18 \mathrm{M}$ sulfuric acid (1 mL of $97 \%$ sulfuric acid diluted to $100 \mathrm{~mL}$ with Milli-Q water) until it falls under that threshold, and then record the dilution and the value of the absorbance. Typically, a dilution factor $(d)$ of 3 is required.

22 Use the data collected from steps 20 and 21, along with the absorptivities for furfural, 5-hydroxymethylfurfural, and acid-soluble lignin $\left(9.7 \pm 0.3 \mathrm{~L} \mathrm{~g}^{-1} \mathrm{~cm}^{-1}, 20.3 \pm 0.4 \mathrm{~L} \mathrm{~g}^{-1} \mathrm{~cm}^{-1}\right.$, and $110 \mathrm{~L} \mathrm{~g}^{-1} \mathrm{~cm}^{-1}$, respectively, at $\left.205 \mathrm{~nm}\right)$, to determine the acid-soluble lignin in the biomass according to Eq. (10). Abbreviations: ASL, acid-soluble lignin; BMB, ball-milled biomass; RBM, raw biomass; $m$ mass; $b$, path length; $\varepsilon$, absorptivity; $d$, dilution factor; HMF, 5-hydroxymethylfurfural; $H$, hydration; $E$, extractives.

$$
\operatorname{ASL}(\%(\mathrm{wt} / \mathrm{wt}))=\left(\frac{\left(A_{A S L}-\varepsilon_{\text {cutuwo }} \times b \times[\text { [urfural }]-\varepsilon_{\text {HMF }} \times b \times[H M F]\right)}{\varepsilon_{A S L} \times b \times m_{B M B} \times\left(1-H_{\text {BMB }}(\%(w t / w t))\right.}\right) \times(1 L) \times d \times\left(1-H_{R B M}(\%(w t / w t))-E_{R B M}(\%(w t / w t))\right) \times 100 \%
$$

23 Remove the filters and filter cakes from the vacuum oven, along with the centrifuge tubes from step 4, and cool them in a vacuum desiccator ( $25 \mathrm{mbar}$ ) for $1 \mathrm{~h}$ at room temperature.

24 Mass the filters and filter cakes and subtract the mass of the filters to determine the Klason lignin content, using Eq. (11). Abbreviations: $m$, mass; CFT, centrifuge tube; $\mathrm{BMB}$, ball-milled biomass; $\mathrm{RBM}$, raw biomass; $H$, hydration; $E$, extractives.

$$
\text { Klason lignin }(\%(\mathrm{wt} / \mathrm{wt}))=\left(\frac{m_{C F T} \& \text { Klason lignin }-m_{C F T}}{m_{\text {Biomass }} \times\left(1-H_{B M B}(\%(w t / w t))\right)}\right) \times\left(1-H_{R B M}(\%(w t / w t))-E_{R B M}(\%(w t / w t))\right) \times 100 \%
$$


Box 5 | Determination of the theoretical monomer yields from the biomass

Procedure

1 Into a 50-mL Parr reactor with a bar-type, PTFE-coated stir bar (20-mm length $\times 10-\mathrm{mm}$ diameter), add the raw biomass $(1 \mathrm{~g})$, ruthenium on carbon ( $5 \%$ (wt/wt), $200 \mathrm{mg}$ ), and methanol $(20 \mathrm{~mL})$.

2 Seal the Parr reactor and then backfill it with $\mathrm{H}_{2}$ gas by filling it with 40 bar of $\mathrm{H}_{2}$ and slowly releasing the pressure.

3 Repeat the backfill for a total of three times.

4 Fill the Parr reactor with 40 bar of $\mathrm{H}_{2}$ gas.

5 Heat the Parr reactor to $250{ }^{\circ} \mathrm{C}$ with stirring for $15 \mathrm{~h}$. Start the timer as soon as the reactor begins heating.

6 Let the Parr reactor cool to room temperature.

7 Release the hydrogen gas and open the Parr reactor.

8 Add $200 \mu \mathrm{L}$ of the $n$-decane stock solution to the reaction solution and stir it with a spatula.

9 Using a 20-mL syringe, withdraw the reaction solution from the Parr reactor.

10 Filter the reaction solution through a syringe filter to remove the catalyst and other insoluble material.

11 Take a sample of the filtrate and inject it onto the gas chromatography instrument using the method described in the Equipment setup: 'Monomer yield quantification using gas chromatography'.

12 Integrate the appropriate peaks and, using the effective carbon number, calculate the yield of the reaction as described in the Equipment setup: 'Monomer yield quantification using gas chromatography', using the effective carbon numbers (ECNs) from Table 8.

13 Data for the biomass types used in this protocol are presented in Table 1.

\section{Biological materials}

$\triangle$ CRITICAL Several different hardwood biomass sources are used as examples in this protocol.

However, to the best of our knowledge, there is no reason why any other biomass source could not be used, with the following exceptions. These sources must not have been heated beyond a temperature of $65^{\circ} \mathrm{C}$ before pretreatment; otherwise, they could suffer from lignin condensation and reduced extraction yields. In addition, the biomass need not be extracted and dried before use, as directed in this procedure. Provided the biomass is sufficiently dry $(\leq 10 \%$ (wt/wt)), the procedures should provide good results. Lignin that is produced from unextracted biomass may contain extractives. If the extractives are undesirable, they can be removed before starting the procedure by using the sequence described in the Reagent setup. If the extractives are of little concern, then proceed without that step. Last, as non-hardwood biomass sources may produce additional or different monomers such as 4-(3-hydroxypropyl)-2-methoxyphenol after hydrogenolysis, be sure to verify the identity of your monomers by gas chromatography-mass spectrometry (GC-MS). $\triangle$ CRITICAL It is important to fully characterize the biomass feedstock before commencing the procedure. We provide experimental procedures to quantify the ash (Box 1); hydration (Box 2); extractives (Box 3); structural sugar (cellulose and hemicellulose); acid-soluble lignin and Klason lignin (Box 4); and lignin monomers (Box 5) of the biomass (see Experimental design for further details).

- Birch wood (2017). This birch wood (Betula pendula) was procured from M. Studer of the Bern University of Applied Sciences. The birch wood was provided as particles of sizes between 1.00 and $3.00 \mathrm{~mm}$.

- Birch wood (2018). This birch wood was procured from M. Studer of the Bern University of Applied Sciences. The birch tree ( 40 years old) was harvested in May of 2018 in Solothurn, Switzerland. The tree was debarked and the stem (trunk) was converted into wood chips and then air-dried at $40{ }^{\circ} \mathrm{C}$ for $24 \mathrm{~h}$. The wood chips were then collected and transported to EPFL, where they were sieved and sorted to remove residual bark and leaves. The wood chips were then milled using a 6-mm screen, and then machine-sieved with a $0.45-\mathrm{mm}$ mesh to remove fines.

- Beech wood (2018). This beech wood (Fagus sylvatica) was procured from M. Studer of the Bern University of Applied Sciences. The beech wood was harvested from Bern, Switzerland, in April of 2018 and air-dried at $40{ }^{\circ} \mathrm{C}$ for $24 \mathrm{~h}$. The wood chips were then collected and transported to EPFL, where they were sieved and sorted to remove residual bark and leaves. The wood chips were then milled using a $6-\mathrm{mm}$ screen, and then machine-sieved with a $0.45-\mathrm{mm}$ mesh to remove fines.

- High-syringyl poplar. This transgenic hybrid poplar with an overexpressed ferulate 5-hydroxylase gene (HSP, Populus tremula $\times$ P. alba, F5H-64, 2014) was procured from R. Meilan of Purdue University. The trees were coppiced in March of 2014. Entire trees were cut near ground level, sawed into 12-inch lengths in the field, and then stored for $1-3$ months in a walk-in freezer $\left(-4{ }^{\circ} \mathrm{C}\right)$ in milk crates. The stems were then oven-dried at $45{ }^{\circ} \mathrm{C}$ for 3-7 $\mathrm{d}$. The wood was then manually debarked using a spokeshave and knife-milled to pass through a $6.35-\mathrm{mm}$ screen by Hazen Research. The milled wood 
was then shipped to EPFL, where it was ball-milled to produce a powder that was used for subsequent experiments.

\section{Reagents}

- 1,4-Dioxane (Carl Roth, cat. no. 4229) !CAUTION 1,4-Dioxane is toxic and highly flammable. Use proper protective equipment and a fume hood while handling it. Ensure that there are no open flames or spark-generating devices nearby while handling this chemical. $\triangle$ CRITICAL 2-Methyltetrahydrofuran (Sigma-Aldrich, cat. no. 155810) can be substituted for 1,4-dioxane during the extraction; however, the amount of hydrochloric acid must be doubled.

- 2-Furfuraldehyde (Acros Organics, cat. no. 18110) ! CAUTION 2-Furfuraldehyde is toxic. Use proper protective equipment and a fume hood while handling it.

- 5-Hydroxymethylfurfural (Sigma-Aldrich, cat. no. H40807)

- Acetone (Thommen-Furler, cat. no. 133-VL54K) $\triangle$ CRITICAL Methanol or ethanol can be substituted for acetone.

- Acetonitrile (Merck, cat. no. 100003) ! CAUTION Acetonitrile is toxic and highly flammable. Use proper protective equipment and a fume hood while handling it. Also, ensure that there are no open flames or spark-generating devices nearby while handling this chemical.

- Cellulases (Novozymes CellicCTec2; Sigma-Aldrich, cat. no. SAE0020)

- Ammonium formate

- Celite

- Chloroform- $d$ ( $\geq 99.8 \mathrm{~d}$-atom\%; Armar, cat. no. 013300) ! CAUTION Chloroform- $d$ is toxic. Use proper protective equipment and a fume hood while handling it.

- Citric acid monohydrate (Sigma-Aldrich, cat. no. C1909)

- Cycloheximide (Sigma-Aldrich, cat. no. C7698) !CAUTION Cycloheximide is toxic. Use proper protective equipment and a fume hood while handling it.

- Decane (TCI Europe NV, cat. no. D0011) ! CAUTION n-Decane is highly flammable. Use proper protective equipment and a fume hood while handling it. Ensure that there are no open flames or spark-generating devices nearby while handling this chemical.

- Dichloromethane (Thommen-Furler, cat. no. 739-VL54K) ! CAUTION Dichloromethane is toxic. Use proper protective equipment and a fume hood while handling it. $\triangle$ CRITICAL Diethyl ether can be substituted for dichloromethane.

- Diethyl ether (stabilized with butylated hydroxytoluene (BHT); Carlo Erba Reagents, cat. no. 528275) ! CAUTION Diethyl ether is highly flammable. Use proper protective equipment and a fume hood while handling it. Ensure that there are no open flames or spark-generating devices nearby while handling this chemical.

- Dimethylsulfoxide- $\mathrm{d}_{6}\left(\mathrm{DMSO}-\mathrm{d}_{6}, \geq 99.8 \mathrm{~d}\right.$-atom\%; Armar, cat. no. 015100)

- Ethanol (Fisher Chemical, cat. no. E/0650DF/15) ! CAUTION Ethanol is toxic and highly flammable. Use proper protective equipment and a fume hood while handling it. Ensure that there are no open flames or spark-generating devices nearby while handling this chemical.

- Ethyl acetate (Thommen-Furler, cat. no. 142-VL54K) !CAUTION Ethyl acetate is extremely flammable. Ensure that there are no open flames or spark-generating devices nearby while handling this chemical.

- Formaldehyde (37\% (wt/wt) solution in water stabilized with methanol (10\% (wt/wt)), Carl Roth, cat. no. 4979) ! CAUTION Formaldehyde is extremely toxic; use proper protective equipment and a fume hood while handling it. $\triangle$ CRITICAL Any other stabilizing group can be substituted for formaldehyde, as indicated in our previous publication ${ }^{24}$. Yields will vary depending on the identity of the stabilizing group and optimization of the protocol for the chosen stabilizing group.

- Hexanes (Thommen-Furler, cat. no. 272-VL54K) ! CAUTION Hexanes are extremely flammable. Ensure that there are no open flames or spark-generating devices nearby while handling this chemical.

- Hydrochloric acid (37\% (wt/wt); Merck, cat. no. 100317) ! CAUTION Hydrochloric acid is extremely corrosive. Use proper protective equipment and a fume hood while handling it.

- Methanol (Thommen-Furler, cat. no. 203-VL54K) !CAUTION Methanol is toxic and highly flammable. Use proper protective equipment and a fume hood while handling it. Ensure that there are no open flames or spark-generating devices nearby while handling this chemical.

- Milli-Q water (18.2 M $\Omega, 0.22-\mu \mathrm{m}$ filtered)

- Propionaldehyde (Acros Organics, cat. no. 220510025) ! CAUTION Propionaldehyde is toxic and flammable. Use proper protective equipment and a fume hood while handling it. Ensure that there are 
no open flames or spark-generating devices nearby while handling this chemical. $\triangle$ CRITICAL Any other stabilizing group can be substituted for propionaldehyde, as indicated in our previous publication $^{24}$. Yields will vary depending on the identity of the stabilizing group and optimization of the protocol for the chosen stabilizing group.

- Ruthenium on carbon (extent of labeling: 5\% (wt/wt) Ru; Sigma-Aldrich, cat. no. 206180) ! CAUTION Ruthenium on carbon is toxic. Use proper protective equipment and a fume hood while handling it.

- Silica gel (SiliaFlash irregular silica gel, 40-63 $\mu \mathrm{m}, 60 \AA$ A SiliCycle, cat. no. R12030B) ! CAUTION Silica gel is known to cause silicosis. Use proper protective equipment and a fume hood while handling it.

- Silica gel with indicator (orange gel, granules $\sim 1-3 \mathrm{~mm}$; Merck, cat. no. 101969)

- Silicone oil (Bluestar Silicones, oil 47V350, viscosity, $350 \mathrm{mPa} \cdot \mathrm{s}$; Silitech, cat. no. 40-131)

- Sodium bicarbonate $\left(\mathrm{NaHCO}_{3}\right.$, Carl Roth, cat. no. 8551)

- Sodium chloride (Carl Roth, cat. no. 9265)

- Sulfuric acid ( $\mathrm{H}_{2} \mathrm{SO}_{4}$; 95-97\% (wt/wt); Merck, cat. no. 100731) ! CAUTION Sulfuric acid is extremely corrosive. Use proper protective equipment and a fume hood while handling it.

- Sulfuric acid $\left(\mathrm{H}_{2} \mathrm{SO}_{4} ; 1 \mathrm{M}\right.$; Honeywell, cat. no. 35276) ! CAUTION Sulfuric acid is extremely corrosive. Use proper protective equipment and a fume hood while handling it.

- Tetracycline (Sigma-Aldrich, cat. no. 87128) ! CAUTION Tetracycline is toxic. Use proper protective equipment and a fume hood while handling it.

- Tetrahydrofuran (stabilized with 0.025\% (wt/wt) BHT; Fisher Chemical, cat. no. T/0701/15) ! CAUTION Tetrahydrofuran is extremely flammable. Ensure that there are no open flames or spark-generating devices nearby while handling this chemical.

- Trisodium citrate dihydrate (Acros Organics, cat. no. 22713)

Gases

- Hydrogen ( $\geq 99.999 \%$ (vol/vol), Alphagaz 1 Hydrogen; Air Liquide (Carbagas), cat. no. P0231L50R2A001) ! CAUTION Hydrogen gas is extremely flammable. Ensure that there are no open flames or sparkgenerating devices nearby while handling this chemical.

- Nitrogen ( $\geq 99.999 \%$ (vol/vol), Alphagaz 1 Nitrogen; Air Liquide (Carbagas), cat. no. P0271L50S2A001)

- Synthetic air ( $\geq 99.999 \%$ ( $\mathrm{vol} / \mathrm{vol}), 20 \pm 2 \%$ ( $20 \mathrm{l} / \mathrm{vol}) \mathrm{O}_{2}$, balance $\mathrm{N}_{2}$; Air Liquide (Carbagas), cat. no. I4520L50R2A001)

\section{Equipment}

\section{Glassware, reactors, and consumables}

- Antistatic gun (Milty, model no. Zerostat 3)

- Bubbler (40 mL; VWR, cat. no. 89063-988)

- Capillary tubes (thin-layer chromatography spotter, both ends open; Marienfeld, cat. no. 2930203 )

- Centrifuge tubes (50 mL; Greiner Bio-One, cat. no. 227261)

- Clamping lid (Plexiglas for 200-mm-diameter test sieves; Fritsch, cat. no. 31.2020.00)

- Dimroth reflux condenser (29/32 joint, 160-mm height, Duran; VWR, cat. no. 210-1681)

- Erlenmeyer flask (500 mL; VWR, cat. no. 214-1133)

- Filter flask, 250 mL (Duran, cat. no. 2120436 5)

- Gas chromatography caps (Infochroma, cat. no. G004-HP-CR-SKFK10)

- Gas chromatography vials (Infochroma, cat. no. G004-HP-H)

- Glass filter funnel (porosity grade 3, $50 \mathrm{~mL}$; Duran, cat. no. $258520 \times 3$ )

- Graduated cylinder (50 mL; Duran, cat. no. 2139017 06)

- High-pressure liquid chromatography caps and vials (Waters, cat. no. 186002640)

- Membrane filtration apparatus (250 mL; Duran, cat. no. 2571054 51)

- Neoprene vacuum adapters (VWR, cat. no. KART420)

- NMR tubes $(400 \mathrm{MHz}, 5 \mathrm{~mm} \times 177.8 \mathrm{~mm}, 0.43-\mathrm{mm}$ wall; Wilmad, cat. no. WG-1228-7)

- Nylon membrane filters $(0.2-\mu \mathrm{m}$ pore size, 47-mm diameter; Whatman, cat. no. 7402-004)

- Oil bath (1 L; Heidolph, cat. no. 504-93000-00)

- pH paper (MColorpHast, pH 0-14; Merck, cat. no. 1.09535)

- Pipette filler (Poulten \& Graf, cat. no. 1 17004)

- Pipette tips (20-200 $\mu \mathrm{L}$ and 100-1,000 $\mu \mathrm{L}$; Tipone, cat. nos. S1111-0706 and S1111-6701)

- Pipette tips (1,000-10,000 $\mu \mathrm{L}$; Semadeni, cat. no. 4973) 
- Polytetrafluoroethylene (PTFE)-coated stir bars (oval, 20-mm length $\times 10$-mm diameter; bar, 30-mm length $\times 10$-mm diameter)

- Quartz-sealable cuvette with septum cap (3.5-mL volume, 10-mm path length, two windows; Hellma Analytics, cat. no. 117-100-10-40)

- Reagent bottle with GL 45 polypropylene cap (500 mL; Simax, cat. no. 1632414321500)

- Round-bottom flasks (29/32, 100, 250, 500, and 1,000 mL; Duran, cat. nos. 24170 27, 24170 37, 24170 46, and 24170 56)

- Sieve pan (stainless steel for 200-mm-diameter test sieves; height: 50 mm; Fritsch, cat. no. 31.1000.03)

- Silica gel chromatography columns (100 g, SNAP Cartridge KP-Sil; Biotage, cat. no. FSK0-1107-0100)

- Spatulas (micro, double, powder; VWR, cat. no. HAMMHSN011-15)

- Syringes (1 mL; Braun, cat. no. 9166106V)

- Syringes (5 and $10 \mathrm{~mL}$; Codan, cat. nos. 62.5607 and 62.6612)

- Syringe filter (Chromafil Xtra H-PTFE 20/25; Macherey-Nagel, cat. no. 729245)

- Test sieve (diameter: $200 \mathrm{~mm}$; height: $32 \mathrm{~mm}$; mesh size: $0.450 \mathrm{~mm}$; VWR, cat. no. 510-0642)

- Test tubes $(16 \times 150 \mathrm{~mm}$; VWR, cat. no. 212-0472)

- Thick-walled glass reactor $(60 \mathrm{~mL}, 150$ p.s.i., 38.1-mm outer diameter, 10.2-cm length, no. 25 frontseal plug; Ace Glass, cat. no. 8648-136)

- Thin-layer chromatography development chamber (Hellendahl staining jar; Biosystems, cat. no. 4200000)

- Thin-layer chromatography plates (aluminum sheets $20 \times 20 \mathrm{~cm}$, TLC Silica gel $60 \mathrm{~F}_{254}$; Merck, cat. no. 1.05554)

- Vacuum desiccator with socket valve (plate diameter: $235 \mathrm{~mm}$; diameter: $320 \mathrm{~mm}$; height: $349 \mathrm{~mm}$; Type 250; VWR, cat. no. 75871-434; containing orange silica gel desiccant)

-Variable-volume single-channel pipettes (0.1-1 mL, 1-10 mL, and 20-200 $\mu \mathrm{L}$; VWR, cat. nos. 613-5265, 613-5267, and 613-5263)

- Volumetric flasks (10, 25, and 500 mL; Duran, cat. nos. 2467110 54, 24671 14 57, and 2467144 54)

\section{Instruments}

- Autoclave (Tuttnauer, model no. 2890 EL/PV-D)

- Automated column machine (Isolera Prime, one-channel, single collection bed, 200-400-nm detector; Biotage, ISO-PSV model)

- Cutting mill (Retsch, model no. SM 200)

- Gas chromatography system (Agilent Technologies, model no. 7890B) with autosampler (model no. 7963) and a flame ionization detector (GC-FID) equipped with an Agilent Technologies HP-5 column (length: $30 \mathrm{~m}$, diameter: $0.320 \mathrm{~mm}$, and film: $0.25 \mu \mathrm{m}$; cat. no. 19091J-413)

- GC-MS-EI system (Agilent Technologies, model no. 7890B) with autosampler (Agilent Technologies, model no. 7963) and mass spectrometer detector with an electron ionization (EI) source (MSD; Agilent Technologies, model no. 5977A) equipped with an Agilent Technologies HP-5MS Ultra Inert column (length: $30 \mathrm{~m}$, diameter: $0.250 \mathrm{~mm}$, and film: $0.25 \mu \mathrm{m}$; cat. no. 19091S-433UI)

- Liquid chromatography (LC) system (Agilent Technologies, model no. 1260 Infinity system) with model no. 1260 high-performance degasser (cat. no. G4225A), 1260 binary pump (cat. no. G1312B), 1260 automated liquid sampler (ALS; cat. no. G1329B), model no. 1260 thermostatted column compartment (TCC; cat. no. G1316A), model no. 1260 diode array detector (DAD; cat. no. G4212B), and model no. 1260 refractive index detector (RID; cat. no. G1362A) equipped with a Phenomenex Luna 5- $\mu \mathrm{m} \mathrm{C18(2)} 100 \AA$ LC column $(150 \mathrm{~mm} \times 4.6 \mathrm{~mm}$; cat. no. 00F-4252-E0) and SecurityGuard Cartridges for C18 guard column $(4 \mathrm{~mm} \times 3.00 \mathrm{~mm}$; cat. no. AJ0-4287)

- LC system (Agilent Technologies, model no. 1260 Infinity system with model no. 1260 high-performance degasser (cat. no. G4225A), model no. 1260 binary pump (cat. no. G1312B), model no. 1260 ALS (cat. no. G1329B), model no. 1260 TCC (cat. no. G1316A), model no. 1260 DAD (cat. no. G4212B), and model no. 1260 RID (cat. no. G1362A) equipped with an Aminex HPX-87H column $(300 \mathrm{~mm} \times 7.8 \mathrm{~mm}$; Bio-Rad, cat. no. 125-0140) and Micro-Guard Cation $\mathrm{H}^{+}$guard column (cat. no. 125-0129))

- High-pressure reactor for hydrogenolysis consisting of a Parr reactor (rated to 200 bar at $350{ }^{\circ} \mathrm{C}$; $50 \mathrm{~mL}$; Hastelloy C-276, moveable head with thermowell, 200-bar pressure gauge with gauge adapter and valve, rupture disk assembly, and double-valve assembly with dip tube; 200-bar rupture disk, PTFE flat gasket; ASME-certified; Parr Instrument, part no. 4792-50 mL-T-HC-VGR-DVD-3000-ASME), hot plate stirrer (Heidolph, cat. no. 505-30000-00-4), thermocouple (K-type; Parr Instrument, cat. no. D002E4), temperature control box (230 volts alternating current (VAC); Omega, cat. no. CN7823), and ceramic band heater (500 W, 230 VAC; Equilabo, cat. no. FOURMICRO2550K) 
- Hot plate stirrer with temperature regulator (Heidolph, cat. no. 505-30081-00-4)

- Microbalance (Ohaus, model. no. AX324)

- Milli-Q Advantage A10 Water Purification System (EMD Millipore, cat. no. Z00Q0V0WW) equipped with a Millipak Express 40 Filter (EMD Millipore, cat. no. MPGP04001), Q-Gard T2 Purification Cartridge (EMD Millipore, cat. no. QGARDT2X1), and Quantum TEX Polishing Cartridge (EMD Millipore, cat. no. QTUM0TEX1)

- Quadrupole time-of-flight (QToF) mass spectrometer (Waters, model no. Xevo G2-S QToF, equipped with electrospray ionization (ESI), atmospheric pressure chemical ionization (APCI), and atmospheric pressure photoionization (APPI) sources)

- Nuclear magnetic resonance spectrometer (Bruker, model no. Avance III 400-MHz, with BBFO-plus probe)

- Rotary evaporator (Hei-VAP Advantage; Heidolph, cat. no. 562-01310-00-1; with Buchi vacuum pump, model no. V-300, with digital interface, model no. I-300; Buchi, cat. no. 11V300220)

- Shaking incubator with 2.5-cm (1-inch) orbit diameter (New Brunswick Scientific, model no. Innova 26)

- Sonicator (USC 300 TH; VWR, cat. no. 142-0084)

- UV-visible scanning spectrophotometer (UV-3100PC; VWR, cat. no. 10037-438)

- Vacuum oven (Vacutherm VT 6025; Thermo Scientific, cat. no. 51014550; with Buchi vacuum pump, model no. V-700)

- Vibratory sieve shaker (Fritsch Analysette)

\section{Reagent setup}

Bulk biomass extractives removal and drying (stock for fractionation)

To remove the extractives from the biomass, follow the steps below:

1 Create $1.5 \mathrm{~L}$ of $80 \%$ (vol/vol) ethanol by mixing $1.2 \mathrm{~L}$ of absolute ethanol with $0.3 \mathrm{~L}$ of Milli-Q water in a 2 -L reagent bottle with a GL 45 polypropylene cap. ! CAUTION Ethanol is toxic and highly flammable. Use proper protective equipment and a fume hood while handling it. Ensure that there are no open flames or spark-generating devices nearby while handling this chemical.

2 Mass $25 \mathrm{~g}$ of biomass into a $500-\mathrm{mL}$ reagent bottle with a GL 45 polypropylene cap.

3 Add $500 \mathrm{~mL}$ of $80 \%$ ethanol to this bottle, cap it, and sonicate it at room temperature $\left(\sim 23-30{ }^{\circ} \mathrm{C}\right)$ for $30 \mathrm{~min}$.

4 Let the solids settle, and then carefully decant the solution.

5 Repeat Steps 3 and 4 twice more with $80 \%$ ethanol, three times with Milli-Q water, and once with absolute ethanol.

6 Loosely cap the $500-\mathrm{mL}$ reagent bottle and place it and the biomass into a vacuum oven at $60{ }^{\circ} \mathrm{C}$ and dry it for at least $16 \mathrm{~h}$ in vacuo ( 50-mbar final pressure).

7 Remove the biomass from the vacuum oven and cool it in a vacuum desiccator ( 25 mbar) for $1 \mathrm{~h}$ at room temperature.

8 Transfer the biomass to an opaque, sealed, storage container, in which it can be stored indefinitely at $23{ }^{\circ} \mathrm{C}$.

$1 \%(w t / w t)(0.1 \mathrm{M})$ sulfuric acid

Add $50 \mathrm{~mL}$ of deionized water to a $100-\mathrm{mL}$ volumetric flask. Tare the flask and add the sulfuric acid $(1.031 \mathrm{~g}, 10.51 \mathrm{mmol})$. Dilute the volume to $100 \mathrm{~mL}$ with deionized water. This solution can be stored at $23{ }^{\circ} \mathrm{C}$ for up to a year. ! CAUTION Sulfuric acid is extremely corrosive. Use proper protective equipment and a fume hood while handling it. !CAUTION This dilution is extremely exothermic. Always add acid to water and not vice versa. Let the solution cool to room temperature before diluting to $100 \mathrm{~mL}$.

\section{n-Decane gas chromatography standard}

Mass the $n$-decane $(400 \mathrm{mg}, 2.81 \mathrm{mmol})$ into a 10 - $\mathrm{mL}$ volumetric flask and dilute the $n$-decane with 1,4-dioxane to $10 \mathrm{~mL}$. This solution can be stored at room temperature for up to 6 months, after which it must be tested for peroxides due to the 1,4-dioxane. If no peroxides have formed, it can be stored for an additional 6 months, after which it must be tested again. ! CAUTION $n$-Decane is highly flammable. Use proper protective equipment and a fume hood while handling it. Ensure that there are no open flames or spark-generating devices nearby while handling this chemical. ! CAUTION 1,4-Dioxane is toxic and highly flammable. Use proper protective equipment and a fume hood while handling it. Ensure that there are no open flames or spark-generating devices nearby while handling this chemical. 


\section{Equipment setup}

\section{Autoclave}

1 Seal all the reagent bottles to be autoclaved with their GL 45 polypropylene caps.

2 Fill one empty reagent bottle with deionized water to roughly the same level as those in the reagent bottles. Leave uncapped.

3 Place all the reagent bottles into the autoclave with the uncapped reagent bottle roughly in the center.

4 Insert the thermocouple(s) into the uncapped reagent bottle.

5 Seal the autoclave.

6 Heat the autoclave to a temperature of at least $121{ }^{\circ} \mathrm{C}$ and a pressure of $220 \mathrm{kPa}(\sim 1 \mathrm{~h})$.

7 Hold the autoclave at that temperature and pressure for $1 \mathrm{~h}$.

8 Let the autoclave slowly cool and exhaust to $90{ }^{\circ} \mathrm{C}$ and $\sim 100 \mathrm{kPa}(\sim 1.5 \mathrm{~h})$.

\section{Automated column machine: silica gel column}

Use the steps below to prepare and run a $100-\mathrm{g}$, silica gel column on an automated column machine (Steps 78 and 79 of the Procedure):

1 Select an appropriately sized silica gel cartridge. Typically, a mass ratio of 1:50 is an advisable starting point, with more difficult separations requiring more silica gel and vice versa. As the automated column machine cartridges typically come in preset sizes, always choose the slightly larger version for initial separation attempts. Here, we use 100-g cartridges because of the size of the sample and the difficulty of the separation.

2 Using the display, program the sequence to include the following phases with the respective column volumes of solvent (solvent required to fill the voids in the packed column being used). For the 100-g cartridge, 1 column volume is $\sim 125 \mathrm{~mL}$ and the flow rate should be $50 \mathrm{~mL} \mathrm{~min}{ }^{-1}$.

- Equilibration at the initial solvent conditions ( 3 column volumes)

- After loading the resting phase at the initial solvent conditions (1 column volume)

- Solvent ramp (10 column volumes)

- Column wash (2 column volumes)

3 Indicate in the method the type of test tube rack being used and fill the test tube rack with test tubes.

4 Set the sample collection to collect all fractions, and enable the UV-visible to observe the 254- and 280-nm bands.

5 Load the silica gel cartridge and start the run sequence. After equilibration, the system will stop and prompt you to load your sample. Press 'load sample' and, using a 10-mL syringe and hexanes, quantitatively transfer the sample to the top of the silica gel cartridge.

6 Restart the run and wait until it is completed.

7 Empty the waste container used to collect any washings and the equilibration solvent.

8 Collect the test tube racks.

\section{Automated column machine: test tube fraction analysis}

Use the thin-layer chromatography (TLC) procedure below to determine which fractions to collect from the racks of test tubes (Step 80 of the Procedure). As the products are not chromophores, a $\mathrm{KMnO}_{4}$ TLC stain will be used to visualize the TLC plates after they have been developed.

1 Take a $20 \times 20$-cm aluminum-backed $\mathrm{SiO}_{2}$ TLC plate and cut it into $5 \times 5$-cm strips. Draw a line in pencil $\sim 0.75 \mathrm{~cm}$ from the bottom of the $5 \times 5$-cm TLC strip. Along this line, draw a perpendicular score every 2-3 $\mathrm{mm}$ and then write an even number below that line from 2 to the number of scores on the strip. Repeat the same procedure on another TLC strip until the highest even number matches the number of test tube fractions.

2 Spot the numbered TLC plates using the TLC spotter with the correspondingly numbered test tube fractions.

3 Place the TLC plate into a TLC chamber containing the solvent mixture specified in the Anticipated results section for the desired isolated compound.

4 The TLC plate will draw solvent up the silica gel through capillary action. Once the solvent line is $\sim 0.75 \mathrm{~cm}$ from the top of the plate, withdraw the plate and use a pencil to mark the point to which the solvent advanced.

5 Air-dry the TLC plate and then place it completely within the $\mathrm{KMnO}_{4}$ TLC stain. This stain requires heat to develop, so place it in an oven $\left(120^{\circ} \mathrm{C}\right)$ for $\sim 1 \mathrm{~min}$. The $\mathrm{KMnO}_{4}$ is purple and it will oxidize any oxidizable chemical species present on the TLC plate, leaving a yellowish-brown spot. 
Table 7 | Effective carbon numbers, retention times, and unreduced molecular weights of the monomers for GC yield calculations using tetrahydrofuran or dioxane as the solvent

\begin{tabular}{lllll} 
Molecule & $\begin{array}{l}\text { Retention time } \\
(\mathbf{m i n})\end{array}$ & $\begin{array}{l}\text { Molecular weight }(\mathrm{g} \\
\left.\mathbf{m o l}^{-1}\right)\end{array}$ & $\begin{array}{l}\text { Unreduced molecular } \\
\text { weight }\left(\mathrm{g} \mathrm{mol}^{-1}\right)\end{array}$ & $\begin{array}{l}\text { Effective } \\
\text { carbon number }\end{array}$ \\
\hline Decane & 5.521 & 142.29 & 142.29 & 10 \\
M1 & 6.935 & 152.19 & 196.20 & 7 \\
M2 & 7.231 & 166.22 & 196.20 & 8 \\
M3 & 7.257 & 166.22 & 196.20 & 8 \\
M4 & 7.517 & 180.25 & 196.20 & 9 \\
M5 & 7.769 & 182.22 & 226.23 & 7 \\
M6 & 7.912 & 196.25 & 226.23 & 8 \\
M7 & 8.019 & 196.25 & 226.23 & 8 \\
M8 & 8.145 & 210.27 & 226.23 & 9 \\
M9 & 8.976 & 212.25 & 226.23 & 7.4 \\
M10 & 8.902 & 226.27 & 226.23 & 8.4 \\
\hline
\end{tabular}

These monomers were observed for the biomass used in this protocol. Other monomers, such as 4-(3-hydroxypropyl)-2-methoxyphenol, may be observed in other biomass sources, especially for biomass sources with a high guaiacyl content.

6 Based on the $R_{\mathrm{f}}$ (fractional distance of elution versus the distance of solvent advance) of the reported compound, narrow down the range of possible fractions and repeat Steps 2-6, but this time running every fraction of interest.

7 Collect the appropriate fractions in a 1-L, 29/32, round-bottom flask.

8 Concentrate the fractions on a rotary evaporator $\left(40{ }^{\circ} \mathrm{C}, 25\right.$-mbar final pressure) to afford the product.

\section{Monomer yield quantification using gas chromatography}

To quantify the yield of monomers from the hydrogenolyses (Steps 117 and 118 of the Procedure), follow the steps below.

1 Run a gas chromatogram using the following parameters:

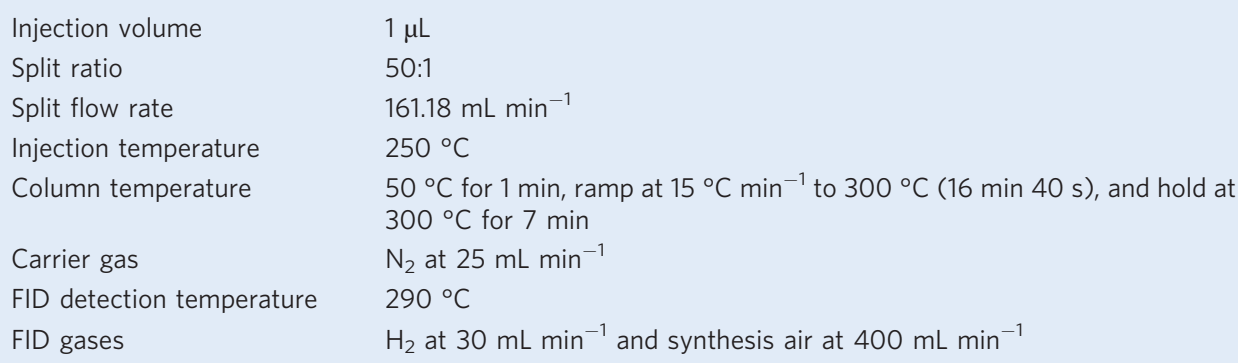

2 Integrate the areas of monomers and decane in the GC-FID chromatogram.

3 Use Eqs. (1-4) to calculate the yield of each monomer on the basis of its integrated area and effective carbon number (ECN). The ECNs of the monomers and decane can be found in Tables 7 and 8 . Abbreviations: $m$, mass; $n$, moles; MW, molecular weight; $A$, area of peak .

$$
\begin{gathered}
n_{\text {decane }}=\frac{m_{\text {decane in sample }}}{\mathrm{MW} \text { decane }} \\
n_{\text {monomer }}=\frac{A_{\text {monomer in sample }}}{A_{\text {decane in sample }}} \times n_{\text {decane }} \times \frac{\mathrm{ECN}_{\text {decane }}}{\mathrm{ECN}_{\text {monomer }}} \\
m_{\text {monomer }}=n_{\text {monomer }} \times \mathrm{MW}_{\text {monomer }} \\
\text { Yield }_{\text {monomer }}=\frac{m_{\text {monomer }}}{m_{\text {biomass }}} \times 100 \%
\end{gathered}
$$


Table 8 | Effective carbon numbers, retention times, and unreduced molecular weights of the monomers for GC yield calculations using methanol as the solvent

\begin{tabular}{llllc} 
Molecule & $\begin{array}{l}\text { Retention time } \\
(\mathrm{min})\end{array}$ & $\begin{array}{l}\text { Molecular weight } \\
\left(\mathrm{g} \mathrm{mol}^{-1}\right)\end{array}$ & $\begin{array}{l}\text { Unreduced molecular } \\
\text { weight }\left(\mathrm{g} \mathrm{mol}^{-1}\right)\end{array}$ & $\begin{array}{l}\text { Effective } \\
\text { carbon number }\end{array}$ \\
\hline Decane & 5.521 & 142.29 & 142.29 & 10 \\
M1 & 6.935 & 152.19 & 196.20 & 8.18 \\
M2 & 7.231 & 166.22 & 196.20 & 9.58 \\
M3 & 7.257 & 166.22 & 196.20 & 9.58 \\
M4 & 7.517 & 180.25 & 196.20 & 10.98 \\
M5 & 7.769 & 182.22 & 226.23 & 8.18 \\
M6 & 7.912 & 196.25 & 226.23 & 9.58 \\
M7 & 8.019 & 196.25 & 226.23 & 9.58 \\
M8 & 8.145 & 210.27 & 226.23 & 10.98 \\
M9 & 8.976 & 212.25 & 226.23 & 8.74 \\
M10 & 8.902 & 226.27 & 226.23 & 10.14 \\
\hline
\end{tabular}

These monomers were observed for the biomass used in this protocol. Other monomers, such as 4-(3-hydroxypropyl)-2-methoxyphenol, may be observed in other biomass sources, especially for biomass sources with a high guaiacyl content.

4 For the yield as a percentage of the Klason lignin content, use Eq. (5). Abbreviations: MKL\%, monomer as a weight percentage $(\%(w t / w t))$ of Klason lignin $(\%(w t / w t))$.

$$
\text { Yield }_{\mathrm{MKL} \%}=\frac{\text { Yield }_{\text {monomer }}}{\text { Klason lignin }(\%(\mathrm{wt} / \mathrm{wt}))} \times 100 \%
$$

C18 reverse-phase chromatography

Use the following parameters for the C18 reverse-phase chromatography of the Procedure (Steps 17 and 56):

Flow rate

Injection volume

Column temperature

Solvent A

Solvent B
$0.7 \mathrm{~mL} \mathrm{~min}{ }^{-1}$

$1 \mu \mathrm{L}$

$25^{\circ} \mathrm{C}$

$\mathrm{pH} 7$ water (ammonium formate in Milli-Q water, $1 \mathrm{mg} \mathrm{mL}^{-1}$ )

Acetonitrile

Run the following gradient:

Time (hours:minutes)
00:00-00:05
$00: 05-00: 20$
$00: 20-00: 25$
$00: 25-00: 35$

Time (hours:minutes)

00:00-00:05

00:25-00:35

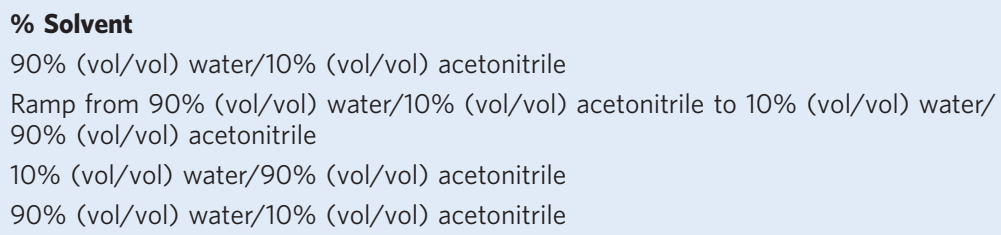

\section{pH 2 aqueous-phase chromatography}

Use the following parameters for the $\mathrm{pH} 2$ aqueous-phase chromatography of the Procedure (Steps 94 and 114):

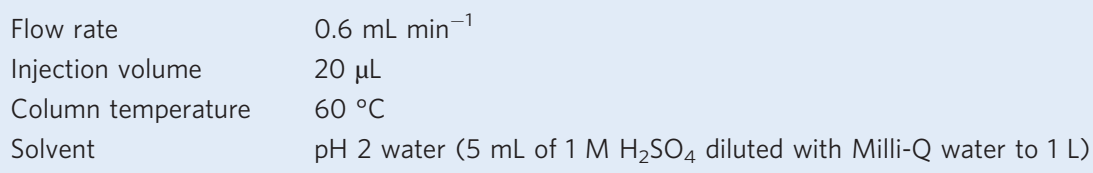

Run the column for $60 \mathrm{~min}$ at $100 \% \mathrm{pH} 2$ water. 
pH 7 aqueous-phase chromatography

Use the following parameters for $\mathrm{pH} 7$ aqueous-phase chromatography (step 20 of the procedure from Box 4: Structural sugar (cellulose and hemicellulose), acid-soluble lignin, and Klason lignin quantification):

$\begin{array}{ll}\text { Flow rate } & 0.6 \mathrm{~mL} \mathrm{~min} \\ \text { Injection volume } & 20 \mu \mathrm{L} \\ \text { Column temperature } & 80{ }^{\circ} \mathrm{C} \\ \text { Solvent } & \mathrm{pH} 7 \text { water (ammonium formate, } 1 \mathrm{mg} \mathrm{mL}^{-1} \text {, in Milli-Q) }\end{array}$

Run the column for $60 \mathrm{~min}$ at $100 \% \mathrm{pH} 7$ water.

Machine sieve

Sieve the biomass using the following procedure to remove fines from it in preparation for its fractionation before the start of the Procedure:

1 Stack the appropriate test sieve $(0.45 \mathrm{~mm})$ onto the bottom sieve pan.

2 Place the biomass to be sieved into the test sieve and then cover it with the clamping lid.

3 Place the assembled sieve stack onto the machine sieve and strap it down.

4 Use the following settings to sieve out any and all fines (diameter $<0.45 \mathrm{~mm}$ ) from the biomass:

$\begin{array}{ll}\text { Pulse } & 5 \text {-s period, } 50 \% \text { duty cycle }(2.5 \mathrm{~s} \text { on; } 2.5 \mathrm{~s} \text { off) } \\ \text { Intensity } & 8 \text { out of } 10 \\ \text { Time } & 30 \mathrm{~min}\end{array}$

5 After running the above sequence, remove the biomass and transfer the sieved materials to separate, appropriate, storage vessels.

\section{${ }^{1}$ H-NMR}

Use the following parameters for the ${ }^{1} \mathrm{H}$-NMR analyses (Steps 34, 77, and 83 of the Procedure):

\begin{tabular}{ll} 
Parameter & Value \\
\hline NS (number of scans) & 16 \\
$D_{1}$ (delay) & $30 \mathrm{~s}$ \\
O1P (transmitter frequency offset) & 6.000 p.p.m. \\
SW (spectral width) & 14.701 p.p.m. \\
DS (dummy scans) & 0
\end{tabular}

\section{${ }^{13} \mathrm{C}-\mathrm{NMR}\left({ }^{1} \mathrm{H}\right.$ decoupled $)$}

Use the following parameters for the ${ }^{13} \mathrm{C}-\mathrm{NMR}$ analyses:

\begin{tabular}{ll} 
Parameter & Value \\
\hline NS (number of scans) & 1,024 \\
$D_{1}$ (delay) & $2 \mathrm{~s}$ \\
O1P (transmitter frequency offset) & $100.000 \mathrm{ppm}$ \\
SW (spectral width) & $236.621 \mathrm{ppm}$ \\
DS (dummy scans) & 4
\end{tabular}

HSQC-NMR $\left({ }^{1} \mathrm{H}-{ }^{13} \mathrm{C}\right.$ multiplicity-edited heteronuclear single-quantum coherence (HSQC) with gradient selection)

Use the following parameters for the HSQC-NMR analyses: 


\begin{tabular}{ll} 
Parameter & Value \\
\hline NS (number of scans) & 32 \\
$D_{1}$ (delays) & $1.5 \mathrm{~s}$ \\
O1P (transmitter frequency offset) & 4.700 p.p.m. \\
SW (spectral width) & 13.1536 p.p.m. \\
DS (dummy scans) & 32
\end{tabular}

\section{Procedure}

$\Delta$ CRITICAL Steps 1-34 describe the procedure for preparing, isolating, and purifying formaldehydestabilized lignin, and Steps 35-83 describe the procedure for preparing, isolating, and purifying propionaldehyde-stabilized lignin. These lignins have different solubilities and provide a different distribution of monomers upon hydrogenolysis.

\section{Formaldehyde biomass fractionation Timing $\sim 10 \mathrm{~h} 5 \mathrm{~min}(\sim 6 \mathrm{~h} 5 \mathrm{~min}$ to isolate only the} formaldehyde-stabilized lignin)

1 Pretreatment of the biomass (Steps 1-4). Mass the extracted and dried biomass (4.5 g) into a 60-mL, thick-walled, glass reactor with an oval PTFE-coated stir bar (20-mm length $\times 10$-mm diameter). (See Fig. 5 for an overview of the formaldehyde fractionation procedure.)

2 Add to the reactor sequentially formaldehyde ( $37 \%$ (wt/wt), $5.2 \mathrm{~mL}, 66 \mathrm{mmol}, 2.6$ equiv.), 1,4 dioxane $(25 \mathrm{~mL})$, and hydrochloric acid (37\% (wt/wt), $2.1 \mathrm{~mL}, 25 \mathrm{mmol}, 1.0$ equiv.).

! CAUTION Formaldehyde is extremely toxic; use proper protective equipment and a fume hood while handling it.

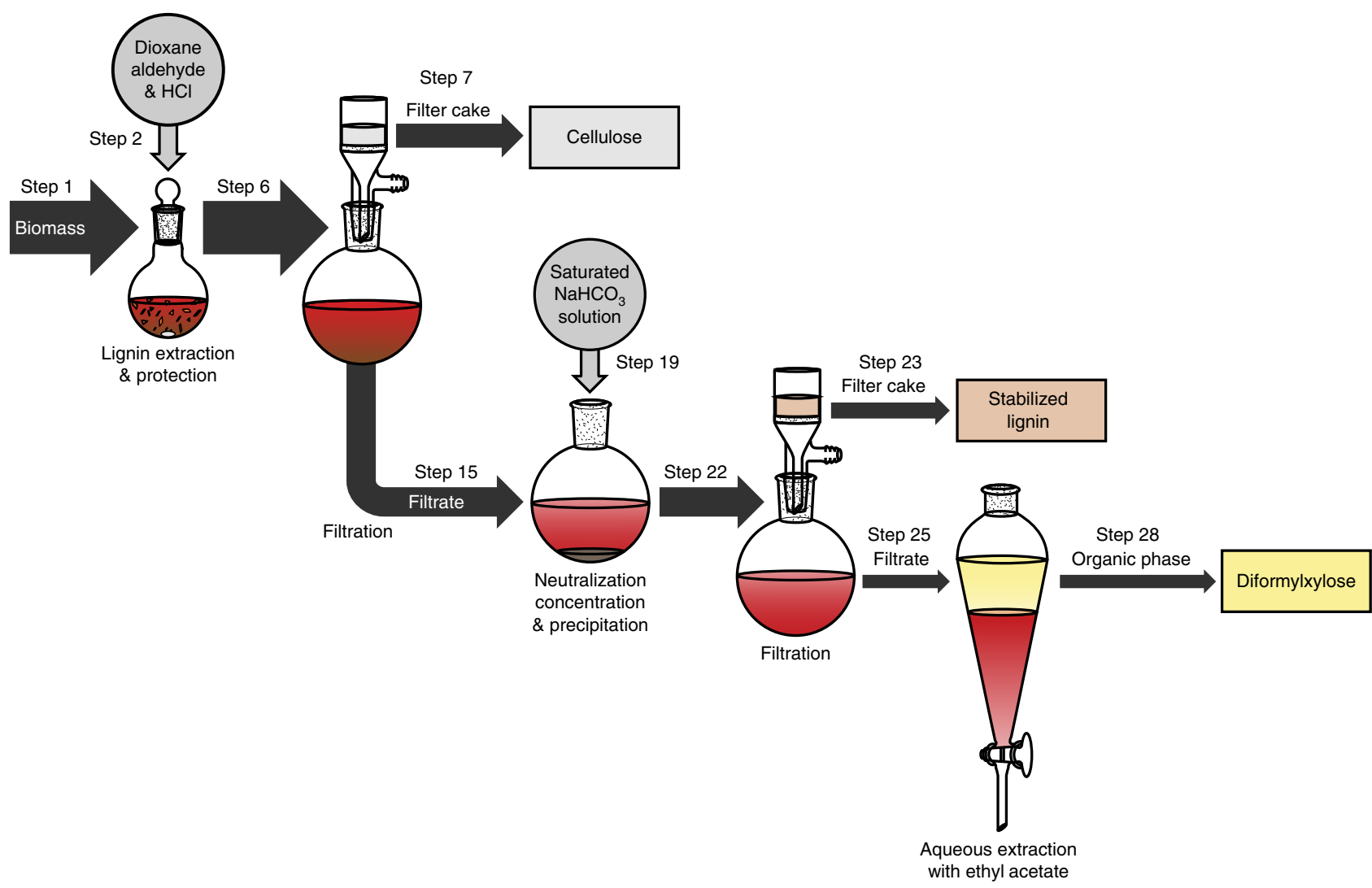

Fig. 5 | The formaldehyde biomass fractionation procedure (Steps 1-34). An overview of the formaldehyde biomass fractionation procedure, which yields cellulose-rich solids, formaldehyde-stabilized lignin, and diformylxylose. The arrow widths are in proportion to the mass of the fraction being isolated for 2018 Birch. Some of the later purification steps have been eliminated for clarity. 
! CAUTION 1,4-Dioxane is toxic and highly flammable. Use proper protective equipment and a fume hood while handling it. Ensure that there are no open flames or spark-generating devices nearby while handling this chemical.

! CAUTION Hydrochloric acid is extremely corrosive. Use proper protective equipment and a fume hood while handling it.

3 Heat the reaction to $95^{\circ} \mathrm{C}$ with stirring for $3.5 \mathrm{~h}$. Swirl the reaction solution every 30 min to ensure homogeneity.

$\triangle$ CRITICAL STEP Incomplete extraction of lignin from the biomass will result if the reaction is not properly stirred.

4 Cool the reaction to room temperature.

\section{? TROUBLESHOOTING}

5 Cellulose collection (Steps 5-14). Assemble a filtration apparatus consisting of a 250-mL filter flask, a neoprene adapter, and a ground-glass-frit Büchner funnel (porosity grade 3 ).

6 Filter the reaction from Step 4 to collect the cellulose, and wash it with dioxane $(2 \times 10 \mathrm{~mL})$ followed by methanol $(2 \times 10 \mathrm{~mL})$ to ensure full extraction of the cellulose, which will have a pink hue. Set the filtrate aside for further processing as detailed in the 'Formaldehyde-stabilized lignin collection' section (Steps 15-23).

! CAUTION Methanol is toxic and highly flammable. Use proper protective equipment and a fume hood while handling it. Ensure that there are no open flames or spark-generating devices nearby while handling this chemical.

7 Transfer the cellulose to a $60-\mathrm{mL}$, thick-walled, glass reactor with an oval PTFE-coated stir bar (20-mm length $\times 10$ - $\mathrm{mm}$ diameter).

8 Add $25 \mathrm{~mL}$ of $1 \%$ (wt/wt) $\mathrm{H}_{2} \mathrm{SO}_{4}$ aqueous solution to the reactor.

9 Heat the reaction to $140{ }^{\circ} \mathrm{C}$ with stirring for $1 \mathrm{~h}$.

10 Assemble a filtration apparatus consisting of a $250-\mathrm{mL}$ filter flask, a neoprene adapter, and a ground-glass-frit Büchner funnel (porosity grade 3).

11 Filter the reaction to collect the cellulose, and wash it with $50 \mathrm{~mL}$ of deionized water followed by $20 \mathrm{~mL}$ of acetone.

! CAUTION Acetone is toxic and highly flammable. Use proper protective equipment and a fume hood while handling it. Ensure that there are no open flames or spark-generating devices nearby while handling this chemical.

12 Transfer the cellulose to a tared, 29/32, 100-mL, round-bottom flask, washing with dichloromethane.

13 Add dichloromethane $(\sim 10 \mathrm{~mL})$ to the flask and then remove the organic solvent in vacuo on a rotary evaporator $\left(40{ }^{\circ} \mathrm{C}\right.$ bath temperature, 300 mbar to $\left.10 \mathrm{mbar}\right)$.

! CAUTION Dichloromethane is highly toxic. Use proper protective equipment and a fume hood while handling it.

14 Re-tare the flask to obtain the mass of the isolated cellulose.

15 Formaldehyde-stabilized lignin collection (Steps 15-23). Transfer the filtrate from Step 6 to a tared, 29/32, 250-mL, round-bottom flask, washing with 1,4-dioxane $(10 \mathrm{~mL})$.

16 Re-mass the round-bottom flask and remove a 1-mL aliquot. Place it into an HPLC vial and cap the vial.

17 Inject the aliquot onto the C18 reverse-phase HPLC column to determine the quantity of 2-furfural and 5-hydroxymethylfurfural produced in the pretreatment reaction (see Equipment setup for column conditions). These data will be relevant for the cellulose and hemicellulose quantifications.

18 Re-mass the round-bottom flask to determine the amount of solution removed.

19 Gradually add saturated $\mathrm{NaHCO}_{3}$ solution $(35 \mathrm{~mL})$ to the filtrate from the previous section and swirl the flask until the acid is neutralized.

! CAUTION This neutralization will result in vigorous bubbling due to the formation of $\mathrm{CO}_{2}$. Proceed with care.

20 Concentrate the solution using a rotary evaporator $\left(35^{\circ} \mathrm{C}\right.$ bath temperature, $60 \mathrm{mbar}$ final pressure). The dioxane will evaporate, causing the formaldehyde-stabilized lignin to precipitate.

21 Assemble a filtration apparatus consisting of a $250-\mathrm{mL}$ filter flask, a neoprene adapter, and a membrane filtration apparatus with a nylon membrane filter.

22 Filter the solution, washing with deionized water $(\sim 50 \mathrm{~mL})$. Let the brown filter cake air-dry for $10 \mathrm{~min}$. Set aside the filtrate for further processing as described in the 'Formylated $\mathrm{C}_{5}$ sugar collection' section (Steps 24-34).

23 Transfer the filter cake to a tared, 29/32, 100-mL, round-bottom flask and dry the filter cake in a vacuum desiccator ( 15 mbar) overnight to afford the formaldehyde-stabilized lignin as a dark-gray or light-brown powder. 
PAUSE POINT Once the lignin is transferred to the desiccator, the fractionation procedure can be paused overnight. Or, if the lignin is the only desired product, discard the filtrate that was set aside in Step 22.

24 Formylated $C_{5}$ sugar collection (Steps 24-34). Transfer the filtrate from Step 22 to a 29/32, 250-mL, round-bottom flask and use a rotary evaporator to concentrate the solution $\left(40{ }^{\circ} \mathrm{C}\right.$ bath temperature, 40 mbar final pressure) to $\sim 50 \mathrm{~mL}$.

25 Transfer the concentrated solution to a $250-\mathrm{mL}$ separatory funnel, washing with ethyl acetate $(5 \mathrm{~mL})$ and deionized water $(5 \mathrm{~mL})$ and dilute it with ethyl acetate $(50 \mathrm{~mL})$.

! CAUTION Ethyl acetate is highly flammable. Ensure that there are no open flames or sparkgenerating devices nearby while handling this chemical.

26 Shake the separatory funnel and separate the layers.

27 Collect the organic layer and return the aqueous fraction to the separatory funnel. Repeat the extraction of the aqueous layer twice more with ethyl acetate $(50 \mathrm{~mL})$.

28 Transfer the organic fractions to a $29 / 32,500-\mathrm{mL}$, round-bottom flask, washing with ethyl acetate $(10 \mathrm{~mL})$.

29 Concentrate the ethyl acetate solution using a rotary evaporator $\left(40{ }^{\circ} \mathrm{C}\right.$ bath temperature, 25 mbar final pressure).

30 Add this concentrated ethyl acetate solution dropwise to a 250-mL Erlenmeyer flask containing $100 \mathrm{~mL}$ of hexanes being stirred at 700 r.p.m. with a bar-type, PTFE-coated stir bar (30-mm length, 10-mm diameter).

! CAUTION Hexanes is toxic and highly flammable. Use proper protective equipment and a fume hood while handling it. Also, ensure that there are no open flames or spark-generating devices nearby while handling this chemical.

31 Assemble a filtration apparatus consisting of a $250-\mathrm{mL}$ filter flask, a neoprene adapter, and a ground-glass-frit Büchner funnel (porosity grade 4).

32 Filter the reaction from Step 30 to remove the insoluble impurities, washing with hexanes $(10 \mathrm{~mL})$.

33 Transfer the filtrate to a tared, $29 / 32,500-\mathrm{mL}$, round-bottom flask, washing with hexanes $(10 \mathrm{~mL})$.

34 Use a rotary evaporator to concentrate the solution $\left(40{ }^{\circ} \mathrm{C}\right.$ bath temperature, $10 \mathrm{mbar}$ final pressure) to afford the diformylxylose as a yellow oil that is $\geq 95 \%$ pure by ${ }^{1} \mathrm{H}$-NMR.

PAUSE POINT Having completed the procedure to this point, the fractionated materials can be stored on the benchtop in sealed vials for at least 3 months before proceeding with the enzymatic cellulose hydrolysis (Steps 84-116) or the lignin hydrogenolysis (Steps 117-128).

\section{Propionaldehyde biomass fractionation $\bigcirc$ Timing $\sim 10 \mathrm{~h} 20 \mathrm{~min}(\sim 6 \mathrm{~h} 40 \mathrm{~min}$ to isolate only} the propionaldehyde-stabilized lignin)

35 Pretreatment of the biomass (Steps 35-40). Mass the extracted and dried biomass (4.5 g) into a 29/32, 100-mL, round-bottom flask containing an oval PTFE-coated stir bar (20-mm length $\times 10$-mm diameter). (See Fig. 6 for an overview of the popionaldehyde fractionation procedure.)

36 Add to the flask sequentially propionaldehyde ( $4.8 \mathrm{~mL}, 67 \mathrm{mmol}, 6.6$ equiv.), 1,4-dioxane (25 mL), and hydrochloric acid (37\% (wt/wt), $0.85 \mathrm{~mL}, 10 \mathrm{mmol}, 1.0$ equiv.).

! CAUTION Propionaldehyde is toxic and highly flammable. Use proper protective equipment and a fume hood while handling it. Ensure that there are no open flames or spark-generating devices nearby while handling this chemical.

! CAUTION 1,4-Dioxane is toxic and highly flammable. Use proper protective equipment and a fume hood while handling it. Also, ensure that there are no open flames or spark-generating devices nearby while handling this chemical,

! CAUTION Hydrochloric acid is extremely corrosive. Use proper protective equipment and a fume hood while handling it.

37 Fit a 29/32 Dimroth condenser onto the flask and connect it to a source of cooling water.

38 Fit a gas bubbler onto the top of the reflux condenser to create an air lock.

$\triangle$ CRITICAL STEP This air lock is essential for the complete extraction of the biomass.

39 Heat the reaction to $85{ }^{\circ} \mathrm{C}$ with stirring for $3 \mathrm{~h}$.

$\triangle$ CRITICAL STEP Incomplete extraction of lignin from the biomass will result if the reaction is not properly stirred.

40 Cool the reaction to room temperature.

\section{? TROUBLESHOOTING}




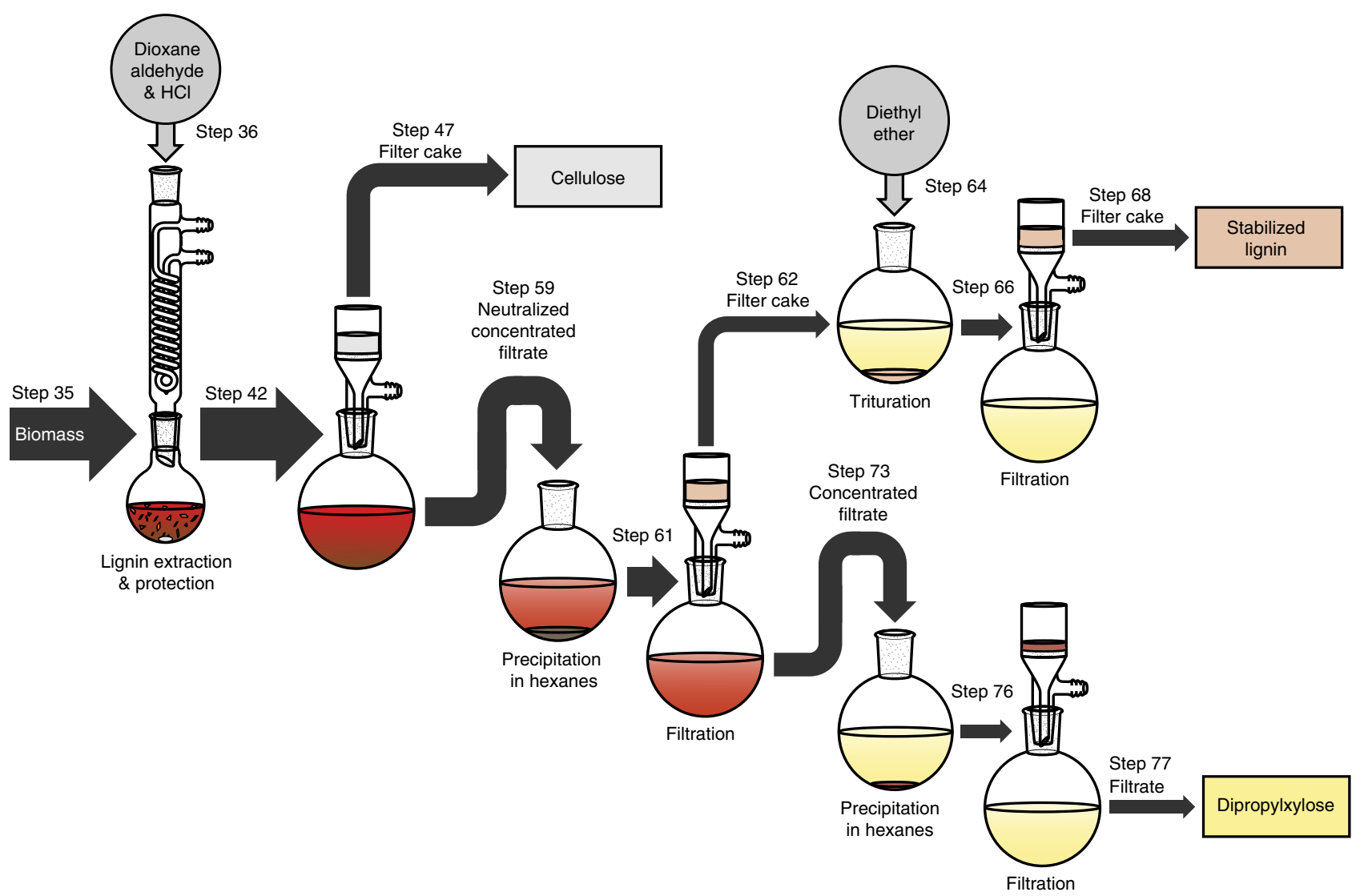

Fig. 6 | The propionaldehyde biomass fractionation procedure (Steps 35-83). An overview of the propionaldehyde biomass fractionation procedure, which yields highly digestible cellulose-rich solids, propionaldehyde-stabilized lignin, and dipropylxylose. The arrow widths are in proportion to the mass of the fraction being isolated for 2018 Birch. Some of the later purification steps for dipropylxylose have been eliminated for clarity.

41 Cellulose collection (Steps 41-49). Assemble a filtration apparatus consisting of a 250-mL filter flask, a neoprene adapter, and a ground-glass-frit Büchner funnel (porosity grade 3 ).

42 Filter the reaction from Step 40 to collect the cellulose, and wash it with dioxane $(2 \times 10 \mathrm{~mL})$ followed by methanol $(2 \times 10 \mathrm{~mL})$ to ensure full extraction of the cellulose, which will have a pink hue.

! CAUTION Methanol is toxic and highly flammable. Use proper protective equipment and a fume hood while handling it. Ensure that there are no open flames or spark-generating devices nearby while handling this chemical.

43 Set the filtrate aside for further processing as detailed in the 'Propionaldehyde-stabilized lignin collection' section (Steps 50-70) and place the Büchner funnel containing the filter cake on another 250-mL filter flask.

44 Without pulling a vacuum, add $20 \mathrm{~mL}$ of saturated sodium bicarbonate solution to the cellulose and stir it with a spatula. The solution will bubble, and the cellulose will turn from a pinkish hue to a light gray.

45 Let the cellulose solution rest for $30 \mathrm{~min}$; then pull a vacuum on the filtration apparatus.

46 Wash the cellulose with $50 \mathrm{~mL}$ of deionized water followed by $20 \mathrm{~mL}$ of acetone.

! CAUTION Acetone is toxic and highly flammable. Use proper protective equipment and a fume hood while handling it. Ensure that there are no open flames or spark-generating devices nearby while handling this chemical.

47 Transfer the cellulose to a tared, 29/32, 100-mL, round-bottom flask, washing with dichloromethane.

48 Add dichloromethane $(\sim 10 \mathrm{~mL})$ to the flask and then remove the organic solvent in vacuo on a rotary evaporator $\left(40{ }^{\circ} \mathrm{C}\right.$ bath temperature, $300 \mathrm{mbar}$ to $\left.10 \mathrm{mbar}\right)$ to afford the cellulose as a lightgray, fibrous material.

49 Re-tare the flask to obtain the mass of the isolated cellulose. 
50 Propionaldehyde-stabilized lignin collection (Steps 50-70). Add $\mathrm{NaHCO}_{3}$ (1.680 g, 20 mmol, 2.0 equiv.) and a bar-type, PTFE-coated stir bar (30-mm length $\times 10-\mathrm{mm}$ diameter $)$ to the filtrate from Step 43.

51 Stir the solution for $30 \mathrm{~min}$ or until the acid is neutralized. If it does not neutralize, add more methanol $(20 \mathrm{~mL})$.

52 Assemble a filtration apparatus consisting of a $250-\mathrm{mL}$ filter flask, a neoprene adapter, and a ground-glass-frit Büchner funnel (porosity grade 3).

53 Filter the reaction from Step 51 to remove the $\mathrm{NaHCO}_{3}$ and $\mathrm{NaCl}$, and then transfer the filtrate to a tared, 29/32, 250-mL, round-bottom flask, washing with 1,4-dioxane (10 mL)

54 Re-mass the round-bottom flask, remove a 1-mL aliquot, place it into an HPLC vial, and cap the vial.

55 Re-mass the round-bottom flask to determine the amount of solution removed.

56 Inject the aliquot set aside onto the C18, reverse-phase HPLC column to determine the quantity of 2-furfural and 5-hydroxymethylfurfural produced in the pretreatment reaction (see Equipment setup for the column conditions). These data will be relevant for the cellulose and hemicellulose quantifications.

57 Use a rotary evaporator to concentrate the solution to remove the methanol $\left(40{ }^{\circ} \mathrm{C}\right.$ bath temperature, 100 mbar final pressure). If a precipitate forms, it is residual $\mathrm{NaHCO}_{3}$ or $\mathrm{NaCl}$ solubilized by the methanol. Filter the reaction, washing with ethyl acetate and using a filtration apparatus consisting of a 250-mL filter flask, a neoprene adapter, and a ground-glass-frit Büchner funnel (porosity grade 3). Transfer the filtrate to a 29/32, 250-mL, round-bottom flask, washing with ethyl acetate, and then concentrate it again using a rotary evaporator $\left(40^{\circ} \mathrm{C}\right.$ bath temperature, 25 mbar final pressure)

58 To the resulting dark-brown oil, add ethyl acetate $(10 \mathrm{~mL})$. The solution should not be viscous and should be easily pipettable.

59 Add the solution dropwise with a pipette (rinsing with $5 \mathrm{~mL}$ of ethyl acetate) to a $500-\mathrm{mL}$ Erlenmeyer flask containing $250 \mathrm{~mL}$ of hexanes being stirred at 700 r.p.m. with a bar-type PTFEcoated stir bar (30-mm length, 10-mm diameter). A reddish-brown precipitate will form.

! CAUTION Ethyl acetate is highly flammable. Ensure that there are no open flames or sparkgenerating devices nearby while handling this chemical.

! CAUTION Hexanes is toxic and highly flammable. Use proper protective equipment and a fume hood while handling it. Also, ensure that there are no open flames or spark-generating devices nearby while handling this chemical.

60 Assemble a filtration apparatus consisting of a 500-mL filter flask, a neoprene adapter, and a membrane filtration apparatus with a nylon membrane filter.

61 Filter the hexanes solution through the filtration apparatus, washing with more hexanes.

62 Collect the filter cake in a tared, 29/32, 250-mL round-bottom flask.

63 Transfer the filtrate to a 29/32, 500-mL round-bottom flask, washing with diethyl ether.

64 Add diethyl ether $(50 \mathrm{~mL})$ to the filter cake and sonicate the solution for $5 \mathrm{~min}$.

! CAUTION Diethyl ether is highly flammable. Ensure that there are no open flames or sparkgenerating devices nearby while handling this chemical.

65 Assemble a filtration apparatus consisting of a 500-mL filter flask, a neoprene adapter, and a membrane filtration apparatus with a nylon membrane filter.

66 Decant the diethyl ether from Step 64 into the filtration apparatus.

67 Add more diethyl ether $(50 \mathrm{~mL})$ to the filter cake; then sonicate it for $5 \mathrm{~min}$, and decant it through the filtration apparatus.

68 Collect any solids that accumulated on the nylon membrane filter and transfer them to the flask containing the residual prior filter cake.

69 Transfer the diethyl ether solution to the flask containing the hexanes and ethyl acetate solution from the earlier precipitation (Step 63).

70 Dry the filter cake in vacuo using a rotary evaporator $\left(40{ }^{\circ} \mathrm{C}\right.$ bath temperature, $25 \mathrm{mbar}$ final pressure) to afford the propionaldehyde-stabilized lignin as a purplish-brown powder.

PAUSE POINT Once the lignin is transferred to the desiccator, the fractionation procedure can be paused overnight. Or, if the lignin is the only desired product, discard the filtrate that was set aside.

71 Propylated $C_{5}$ sugar collection (Steps 71-83). Concentrate the hexanes, ethyl acetate, and diethyl ether solution from Step 69 in vacuo on a rotary evaporator $\left(40{ }^{\circ} \mathrm{C}\right.$ bath temperature, $25 \mathrm{mbar}$ final pressure). 
72 To the resulting dark-brown oil, add diethyl ether $(10 \mathrm{~mL})$. The solution should not be viscous and should be easily pipettable.

73 Using a pipette, add this solution dropwise (rinsing with $5 \mathrm{~mL}$ of diethyl ether) to a $500-\mathrm{mL}$ Erlenmeyer flask containing $250 \mathrm{~mL}$ of hexanes being stirred at 700 r.p.m. with a bar-type, PTFEcoated stir bar (30-mm length, 10-mm diameter).

74 Add activated carbon $(1 \mathrm{~g})$ to the filter flask. Stir for $10 \mathrm{~min}$.

75 Assemble a filtration apparatus consisting of a 500-mL filter flask, a neoprene adapter, and a groundglass-frit Büchner funnel (porosity grade 3). Create a 1-cm pad of vacuum-and-hand-compressed Celite in the ground-glass-frit Büchner funnel.

76 Filter the hexanes solution from Step 74 through the filtration apparatus, washing with more hexanes $(25 \mathrm{~mL})$.

77 Transfer the filtrate to a $29 / 32,500-\mathrm{mL}$, round-bottom flask, washing with hexanes, and concentrate in vacuo on a rotary evaporator $\left(40{ }^{\circ} \mathrm{C}, 25 \mathrm{mbar}\right.$ final pressure). The resulting yellow oil should be $\geq 70 \%$ (wt/wt) dipropylxylose by ${ }^{1} \mathrm{H}-\mathrm{NMR}$, assuming that the impurities are largely alkyl in nature $\left(\mathrm{R}-\mathrm{CH}_{2}-\mathrm{R}\right)$. To purify the dipropylxylose further, follow Steps 78-83.

78 Prepare a 100-g, silica gel column on an automated column machine, using a hexanes:ethyl acetate gradient with an initial solvent ratio of 94:6, which increases over 10 column volumes to 50:50. For a detailed description of how to run such a column, see the Equipment setup: 'Automated column machine' sections.

! CAUTION Silica gel is known to cause silicosis. Use proper protective equipment and a fume hood while handling it.

79 Once the column has equilibrated, load the yellow oil from Step 77 onto the column, washing with hexanes, and then run the programmed sequence, collecting all the fractions.

80 Transfer the appropriate fractions-as determined by comparison of the $R_{\mathrm{f}}$ values of their contents with that provided for dipropylxylose in the Anticipated results section-to a 29/32, 500-mL, roundbottom flask. Two diastereomers of the dipropylxylose will be produced.

81 Concentrate the collected fractions using a rotary evaporator $\left(40^{\circ} \mathrm{C}, 25 \mathrm{mbar}\right.$ final pressure).

82 Transfer the resulting yellow oil to a tared, 29/32, 100-mL, round-bottom flask, washing with ethyl acetate.

83 Concentrate the resulting solution using a rotary evaporator $\left(40^{\circ} \mathrm{C}, 10 \mathrm{mbar}\right.$ final pressure $)$ to afford the dipropylxylose as a mixture of diastereomers. The diastereomers of dipropylxylose can be separated, but it takes multiple silica gel columns, along with the cutting and pooling of fractions, to achieve the separation.

DAUSE POINT Having completed the Procedure to this point, the fractionated materials can be stored on the benchtop in sealed vials for at least 3 months before proceeding with enzymatic cellulose hydrolysis (Steps 84-116) or lignin hydrogenolysis (Steps 117-128).

\section{Enzymatic cellulose hydrolysis Timing $\sim 78$ h $40 \mathrm{~min}$}

$\triangle$ CRITICAL This procedure is used to determine the yield of glucose one would obtain from the enzymatic hydrolysis (Steps 84-95) of the celluloses isolated in either the formaldehyde biomass fractionation procedure (Step 14) or the propionaldehyde biomass fractionation procedure (Step 49).

As complete enzymatic hydrolysis of the celluloses may not occur, a compositional analysis procedure (Steps 96-116) is also described so that the extent of the hydrolysis can be ascertained.

84 Enzymatic hydrolysis of the cellulose (Steps 84-95). Prepare $50 \mathrm{~mL}$ of a $0.1 \mathrm{M}, \mathrm{pH} 5$, citrate buffer by diluting trisodium citrate dihydrate $(956 \mathrm{mg}, 3.25 \mathrm{mmol})$ and citric acid monohydrate $(368 \mathrm{mg}$, $1.75 \mathrm{mmol}$ ) to $50 \mathrm{~mL}$ with Milli-Q water in a 50 -mL volumetric flask.

85 Prepare a tetracycline stock solution by first dissolving the tetracycline $(20 \mathrm{mg}, 0.045 \mathrm{mmol})$ in $1.4 \mathrm{~mL}$ of absolute ethanol, and then diluting the resulting solution with $0.6 \mathrm{~mL}$ of Milli-Q water in a 5 -mL vial with screw cap.

! CAUTION Tetracycline is toxic. Use proper protective equipment and a fume hood while handling it. ! CAUTION Ethanol is toxic and highly flammable. Use proper protective equipment and a fume hood while handling it. Ensure that there are no open flames or spark-generating devices nearby while handling this chemical.

86 Prepare a cycloheximide stock solution by combining cycloheximide ( $20 \mathrm{mg}, 0.071 \mathrm{mmol})$ with $2 \mathrm{~mL}$ of Milli-Q water in a 5-mL vial with screw cap.

! CAUTION Cycloheximide is toxic. Use proper protective equipment and a fume hood while handling it. 
87 Mass $300 \mathrm{mg}$ of cellulose from Step 14 or 49 into each of three 20-mL glass vials with PTFE-coated stir bars.

88 To each vial, via a pipette, add $11.3 \mathrm{~mL}$ of the citrate buffer, $0.4 \mathrm{~mL}$ of the tetracycline solution, and $0.3 \mathrm{~mL}$ of the cycloheximide solution.

89 Cap the vials and place them in a shaking incubator at 250 r.p.m. and $50{ }^{\circ} \mathrm{C}$ for $1 \mathrm{~h}$.

90 Remove the vials and add 10 filter paper units (FPU) of cellulases.

91 Return the vials to the incubator at 250 r.p.m. and $50{ }^{\circ} \mathrm{C}$, and continue to heat and shake them for $72 \mathrm{~h}$.

92 Let the vials cool to room temperature.

93 Transfer the contents of the vials to $50-\mathrm{mL}$ volumetric flasks, washing with Milli-Q water, and dilute to $50 \mathrm{~mL}$ with Milli-Q water.

94 Shake the flasks and let any solids settle; then remove a 1-mL aliquot and filter it through a syringe filter into an HPLC vial.

95 Cap the vial and inject the solution onto the pH 2 aqueous-phase HPLC column to determine the glucose concentration; use this value to determine the yield of glucose, taking into account the hydration of the cellulose (see Equipment setup (for the column conditions) and the 'Determination of the cellulose hydration' section below).

? TROUBLESHOOTING

96 Determination of the cellulose hydration (Steps 96-99). Mass $300 \mathrm{mg}$ of cellulose from Step 14 or 49 into each of three tared, $50-\mathrm{mL}$, self-standing centrifuge tubes.

97 Lightly cap the centrifuge tubes and place them into a vacuum oven at $60{ }^{\circ} \mathrm{C}$ and dry them for at least $16 \mathrm{~h}$ in vacuo ( $50 \mathrm{mbar}$ final pressure).

98 Remove the centrifuge tubes from the vacuum oven and cool them in a vacuum desiccator ( 25 mbar $)$ at room temperature for an hour.

99 Re-mass the centrifuge tubes and calculate the mass loss. Use Eq. (7) from Box 2 to calculate the hydration of the cellulose.

100 Determination of the glucose content of the cellulose (Steps 100-116). Prepare $25 \mathrm{~mL}$ of $72 \%$ (wt/wt) $\mathrm{H}_{2} \mathrm{SO}_{4}$ (specific gravity $=1.634 \mathrm{~g} \cdot \mathrm{mL}^{-1}$ ) by adding $30.97 \mathrm{~g}$ of concentrated sulfuric acid to $8 \mathrm{~g}$ of deionized water in a $25-\mathrm{mL}$ volumetric flask and then diluting with deionized water to a final solution volume of $25 \mathrm{~mL}$.

! CAUTION This dilution is extremely exothermic. Always add acid to water and not vice versa. Let the solution cool to room temperature before diluting to $25 \mathrm{~mL}$.

! CAUTION Sulfuric acid is extremely corrosive. Use proper protective equipment and a fume hood while handling it.

101 Into three, separate, new, $50-\mathrm{mL}$, self-standing centrifuge tubes, add a $0.2-\mu \mathrm{m}$ nylon membrane filter.

102 Place the centrifuge tubes from Step 101, loosely capped, into the vacuum oven at $60{ }^{\circ} \mathrm{C}$ and leave until Step 110.

103 Add oval stir bars $(20-\mathrm{mm}$ long $\times 10$ - mm diameter $)$ to the three centrifuge tubes from Step 99, which contain the dried cellulose. If one would prefer to start with fresh cellulose, mass $300 \mathrm{mg}$ of cellulose from Step 14 or 49 into each of three tared, $50-\mathrm{mL}$, self-standing centrifuge tubes.

104 Into each of the centrifuge tubes from Step 104, add $7.5 \mathrm{~mL}$ of $72 \%$ (wt/wt) (12 M) $\mathrm{H}_{2} \mathrm{SO}_{4}$.

105 Cap the centrifuge tubes, shake, and vortex them to distribute the solid; then sonicate them for $2 \mathrm{~h}$ at $30^{\circ} \mathrm{C}$.

106 Transfer the contents of the centrifuge tubes to $500-\mathrm{mL}$ reagent bottles with GL 45 polypropylene caps and dilute the solutions to $\sim 250 \mathrm{~mL}$ with Milli-Q water.

107 Autoclave the bottles for $1 \mathrm{~h}$ at $120^{\circ} \mathrm{C}$.

108 Transfer the hot solutions $\left(\sim 85^{\circ} \mathrm{C}\right)$ to a refrigerator and let them cool.

!CAUTION These solutions will be extremely hot.

PAUSE POINT After the solutions have cooled, one can either directly proceed with Steps 109-116 or pause the procedure for the day. It is recommended that one wait until the next day, given the time it takes to run Steps 104-108 and because it gives more time for the nylon filters in the centrifuge tubes from Step 101 to fully dry in the vacuum oven.

109 Remove the centrifuge tubes containing the nylon membrane filters from Step 103 from the vacuum oven and cool them in a vacuum desiccator ( $25 \mathrm{mbar})$ for $1 \mathrm{~h}$ at room temperature.

110 Mass the centrifuge tubes from Step 110 and record the mass.

111 Remove the reagent bottles from the refrigerator and filter the solutions through the dried, tared, $0.2-\mu \mathrm{m}$ nylon membrane filters contained in the centrifuge tubes from Step 110, washing with Milli-Q water. 
112 Place the nylon membrane filters and filter cakes into their corresponding centrifuge tubes from Step 110 and loosely cap the centrifuge tubes. Place them in a vacuum oven at $60{ }^{\circ} \mathrm{C}$ and dry them for $24 \mathrm{~h}$ in vacuo ( $\sim 50 \mathrm{mbar}$ final pressure). If there is residual precipitate adhered to the walls of the filtration apparatus after the filtration, wash it into the centrifuge tubes with ethanol.

113 Transfer the filtrates to separate $500-\mathrm{mL}$ volumetric flasks, diluting with Milli-Q water; then return the filtrates to the $500-\mathrm{mL}$ reagent bottles.

114 Remove $1 \mathrm{~mL}$ from each of the $500-\mathrm{mL}$ reagent bottles and filter it through a syringe filter into an HPLC autosampler vial. Label and cap the HPLC vial, and then inject it onto the pH 2 HPLC column to determine the concentrations of glucose, xylose, 5-hydroxymethylfurfural, and 2-furfural in the sample (Equipment setup: 'pH 2 aqueous-phase chromatography'). When presenting the data, add the HPLC responses (grams per liter) of 5-hydroxymethylfurfural and 2-furfural reconstituted as glucose (multiply the 5-hydroxymethylfurfural response by 1.43) and xylose (multiply the 2-furfural response by 1.56) to the observed yields for those of glucose and xylose. Use Eq. (9) from Box 4 while excluding the hydration and extractives terms to calculate the contribution of each sugar to the overall mass of the material.

115 Remove the filters and filter cakes from the vacuum oven, along with their centrifuge tubes from Step 112, and cool them in a vacuum desiccator ( 25 mbar) for $1 \mathrm{~h}$ at room temperature.

116 Mass the filters and filter cakes and subtract the mass of the filters to determine the mass of Klason lignin. Use Eq. (11) from Box 4 while exluding the hydration and extractives terms to calculate the contribution of the Klason lignin to the overall mass of the material.

\section{Lignin hydrogenolysis Timing $\sim 6 \mathrm{~h} 40 \mathrm{~min}$ for formaldehyde-stabilized lignin or $5 \mathrm{~h}$ $40 \mathrm{~min}$ for propionaldehyde-stabilized lignin \\ $\triangle$ CRITICAL This procedure describes the depolymerization by hydrogenolysis of the aldehyde-} stabilized lignin isolated from either the formaldehyde biomass fractionation procedure (Step 23) or the propionaldehyde biomass fractionation procedure (Step 70). The yield of monomers that is obtained from these procedures, as determined by gas chromatography, is used to determine the quality of the lignin that was extracted from the raw lignocellulosic biomass by comparing it to the yield obtained for the direct hydrogenolysis of that same biomass using the procedure described in Box 5: Determination of the theoretical monomer yields from the biomass.

117 Add the stabilized lignin (200 mg), ruthenium on carbon (5\% (wt/wt), $100 \mathrm{mg}$ ), and tetrahydrofuran $(20 \mathrm{~mL})$ to a $50-\mathrm{mL}$ Parr reactor with a bar-type PTFE-coated stir bar (20-mm length $\times 10$-mm diameter).

! CAUTION Tetrahydrofuran is toxic and highly flammable. Use proper protective equipment and a fume hood while handling it. Ensure that there are no open flames or spark-generating devices nearby while handling this chemical.

! CAUTION Ruthenium on carbon is toxic. Use proper protective equipment and a fume hood while handling it.

118 Seal the Parr reactor and then backfill it with $\mathrm{H}_{2}$ gas by filling it with 40 bar of $\mathrm{H}_{2}$ and slowly releasing the pressure.

! CAUTION Hydrogen gas is highly flammable. Use proper protective equipment and a fume hood while handling it. Ensure that there are no open flames or spark-generating devices nearby while handling this chemical.

! CAUTION High-pressure gas is in use. Use proper protective equipment and appropriate equipment for filling and running the reaction.

119 Repeat the backfill for a total of three times.

120 Fill the Parr reactor with 40 bar of $\mathrm{H}_{2}$ gas.

121 Heat the Parr reactor to $250{ }^{\circ} \mathrm{C}$ with stirring for $4 \mathrm{~h}$ for formaldehyde-stabilized lignin (from Step 23) and for $3 \mathrm{~h}$ for propionaldehyde-stabilized lignin (from Step 70). Start the timer as soon as the reactor begins heating.

! CAUTION The reactor will be extremely hot. Handle with care.

$\triangle$ CRITICAL STEP At $250{ }^{\circ} \mathrm{C}$, lower monomer yields may result through degradation or overreduction if the reaction is left longer than the prescribed amount of time. Reaction temperatures as low as $175{ }^{\circ} \mathrm{C}$ can be used; however, more time will be required to convert the lignin ( $>12 \mathrm{~h}$ ). Here, we present optimal conditions for the determination of the monomer yield from the stabilized lignin for the biomass sources used in this paper.

122 Let the Parr reactor cool to room temperature. 
123 Release the hydrogen gas and open the Parr reactor.

124 Add $200 \mu \mathrm{L}$ of the $n$-decane stock solution to the reaction solution and stir it with a spatula. ! CAUTION The $n$-decane stock solution is toxic and highly flammable. Use proper protective equipment and a fume hood while handling it. Ensure that there are no open flames or sparkgenerating devices nearby while handling this chemical.

125 Using a $20-\mathrm{mL}$ syringe, withdraw the reaction solution from the Parr reactor.

126 Filter the reaction solution through a syringe filter to remove the catalyst.

127 Take a sample of the filtrate and inject it onto the gas chromatography instrument, using the method described in Equipment setup: 'Monomer yield quantification using gas chromatography'.

128 Integrate the appropriate peaks and, using the effective carbon number, calculate the yield of the reaction, as described in Equipment setup: 'Monomer yield quantification using gas chromatography'. ? TROUBLESHOOTING

\section{Troubleshooting}

Troubleshooting advice can be found in Table 9.

\section{Table 9 | Troubleshooting table}

\begin{tabular}{lll} 
Step & Problem & Possible reason \\
\hline 4 and 40 & $\begin{array}{l}\text { Incomplete lignin } \\
\text { extraction }\end{array}$ & $\begin{array}{l}\text { The acid concentration is insufficient for scission of } \\
\text { the lignin-carbohydrate bonds }\end{array}$
\end{tabular}

The reaction rate of lignin-carbohydrate bond cleavage is slow

The reaction time was too short for complete lignin-carbohydrate bond cleavage

$95 \quad$ Low enzymatic hydrolysis yield

Low monomer yields from the isolated lignin hydrolysis yields
There is still a substantial quantity of lignin in the cellulose

If the hydration of the cellulose falls below $\sim 2 \%$ (wt/wt), the enzymatic hydrolysis of the cellulose may give low yields due to collapse of the pore structure

If the sequence involving the sodium bicarbonate or dilute sulfuric acid wash of the cellulose is not performed, the yield of glucose monomers from the cellulose will suffer, regardless of the pretreatment methodology, because of the presence of aldehyde species bound to the cellulose surface

The $\mathrm{pH}$ of the enzymatic hydrolysis is not 5. Enzymes are highly susceptible to variations in $\mathrm{pH}$, and small deviations from the optimal $\mathrm{pH}$ can cause poor

The cellulases are no longer viable. They can degrade over time, especially if stored improperly

Lignin in the native biomass is already condensed. If the biomass is dried at a temperature that exceeds $65^{\circ} \mathrm{C}$, the lignin can undergo degradation, resulting in a reduced yield of monomers from the extracted material

The biomass used in the extraction is a poor source of uncondensed lignin. Some sources of biomass simply do not provide high (i.e., $>40 \%$ (wt/wt) versus Klason lignin) yields of monomers from lignin

\section{Solution}

Up to 4.5-fold more acid can be used without detriment to the procedure. When using more acid, be sure to adjust the quantity of base used during the neutralization step accordingly

Reaction temperatures of up to $100^{\circ} \mathrm{C}$ can be used for the extraction protocol. The optimal temperatures for the biomasses used in this paper were described in the procedures, but higher temperatures can be used

Extraction times of up to $5 \mathrm{~h}$ can be used with limited detriment to the products of the extraction

Repeat the extraction procedure, taking into consideration the advice provided in this section regarding incomplete lignin extraction

Repeat the extraction procedure and perform the final evaporation sequence at $25^{\circ} \mathrm{C}$ and $100 \mathrm{mbar}$

Wash the cellulose again with the saturated sodium bicarbonate solution or sulfuric acid solution, followed by deionized water and acetone

Repeat the procedure and adjust the $\mathrm{pH}$ to 5 by using citric acid or sodium citrate after adding the cellulose

Run a control experiment using Avicel PH-101 cellulose or another commercially available high-purity cellulose to determine the viability of the cellulases

Decrease the temperature used to dry the biomass to $<65^{\circ} \mathrm{C}$

Perform a direct hydrogenolysis on the material as described in Box 5 to determine the potential yield of monomers from the material. If the yield is low, the lignin probably has many native interunit $\mathrm{C}-\mathrm{C}$ bonds and will provide a low yield of monomers post separation. If this is the case, replace the biomass source

Table continued 


\section{Table 9 (continued)}

\begin{tabular}{ll} 
Step Problem & Possible reason \\
\hline & The lignin did not depolymerize completely or the \\
& product monomers degraded. The hydrogenolysis can \\
proceed rapidly, depending on the temperature of the & hydrogenolysis, as well as the quality of the lignin that is \\
& extracted. If the extracted lignin is free of sugars or \\
& other by-products, the hydrogenolysis could be \\
& completed in as little as $1 \mathrm{~h}$ at $250^{\circ} \mathrm{C}$. During the \\
& remaining reaction time, the monomers that were \\
& produced can degrade under the hydrogenolysis \\
& conditions. In general, reaction temperatures between \\
& 175 and $250{ }^{\circ} \mathrm{C}$ are required to convert the lignin, with \\
& lower temperatures requiring more time to fully convert \\
& Too much or too little catalyst was added to the \\
& hydrogenolysis. If there is too much catalyst in the \\
& hydrogenolysis, the monomers will be over-reduced \\
& and may degrade, resulting in reduced yields. A similar \\
& result will occur if there is too little catalyst, as the \\
& reaction will be incomplete
\end{tabular}

\section{Solution}

Decrease the reaction time from $3 \mathrm{~h}$ to $2 \mathrm{~h}$. If that does not work, increase the time to $\geq 6 \mathrm{~h}$. If that still does not work, reduce the temperature to $200{ }^{\circ} \mathrm{C}$ and let the reaction run overnight $(>12 \mathrm{~h}$ )

This variable often works in concert with the reaction time; try re-subjecting the reaction solution to the hydrogenolysis for a further $3 \mathrm{~h}$. If the yields improve, there is probably not enough catalyst in the reaction. Conversely, if the yields degrade or do not improve, there could be too much catalyst. Try repeating the hydrogenolysis with a lower loading of catalyst

\section{Timing}

Steps 1-4, pretreatment of the biomass: $\sim 4$ h $35 \mathrm{~min}$

Steps 5-14, cellulose collection: $\sim 2$ h $50 \mathrm{~min}$

Steps 15-23, formaldehyde-stabilized lignin collection: $\sim 1 \mathrm{~h} 40 \mathrm{~min}$

Steps 24-34, formylated $\mathrm{C}_{5}$ sugar collection: $\sim 2 \mathrm{~h} 5 \mathrm{~min}$

Steps 35-40, pretreatment of the biomass: $\sim 4$ h $20 \mathrm{~min}$

Steps 41-49, cellulose collection: $\sim 1$ h 25 min

Steps 50-59, propionaldehyde-stabilized lignin collection: $\sim 2$ h $30 \mathrm{~min}$

Steps 60-83, propylated $\mathrm{C}_{5}$ sugar collection: $\sim 3 \mathrm{~h} 15 \mathrm{~min}$

Steps 84-95, enzymatic hydrolysis of the cellulose: $\sim 78 \mathrm{~h} 40 \mathrm{~min}$

Steps 96-99, determination of the cellulose hydration: $\sim 17$ h $30 \mathrm{~min}$

Steps 100-116, determination of the glucose content of the cellulose: $\sim 38$ h 20 min

Steps 117-128, lignin hydrogenolysis: $\sim 6$ h $40 \mathrm{~min}$ for formaldehyde-stabilized lignin or $5 \mathrm{~h} 40 \mathrm{~min}$ for propionaldehyde-stabilized lignin

The overall timings for Steps 1-34, 35-83, and 84-116 are lower than the sum of the timings here because many of the steps in those sequences can be performed simultaneously. For example, the enzymatic hydrolysis of cellulose requires $72 \mathrm{~h}$ of reaction, during which the other steps of that sequence can be performed.

\section{Anticipated results}

\section{Formaldehyde biomass fractionation protocol}

After completion of this procedure (Steps 1-34), we anticipate the collection of three separate biomass fractions: cellulose-rich solids, formaldehyde-stabilized lignin, and diformylxylose. For birch wood, we expect that the cellulose-rich solids will appear as a fluffy, beige, fibrous powder (Step 14) representing $43.0 \%$ (wt/wt) (2.1373 g) of the raw, unextracted biomass. Enzymatic hydrolysis (Steps $84-95)$ of this cellulose will yield $33.8 \%$ (wt/wt) as glucose and 3.6\% (wt/wt) as xylose, representing $45.2 \mathrm{~mol} \%$ of the glucan and $8.7 \mathrm{~mol} \%$ of the xylan, respectively, in the raw biomass (note: the weight per weight percentages of glucose and xylose were calculated as the dehydrated glucan and xylan, respectively). The formaldehyde-stabilized lignin will be isolated as a gray powder (Step 23), representing $24.0 \%$ (wt/wt) (1.2150 g) of the raw, unextracted biomass after correcting for the formaldehyde stabilization. Hydrogenolysis of this powder (Steps 117-128) will yield 34\% (wt/wt) as monomers (after correction for hydrodeoxygenation), for an overall yield of monomers of $8.57 \%$ (wt/wt) versus dry biomass (8.01\% (wt/wt) versus the raw, unextracted biomass). The diformylxylose will be isolated as a yellow oil (Step 34), representing 13.2\% (wt/wt) (0.8631 g, corrected for the formaldehyde stabilization and converted to xylan) of the raw, unextracted biomass and $74 \mathrm{~mol} \%$ of the xylan. 
For beech wood, we expect that the cellulose-rich solids will appear as a fluffy, beige, fibrous powder (Step 14), representing 35.3\% (wt/wt) (1.7548 g) of the raw, unextracted biomass. Enzymatic hydrolysis (Steps $84-95$ ) of this cellulose will yield $40.5 \%$ (wt/wt) as glucose and $4.1 \%$ (wt/wt) as xylose, representing $43.1 \mathrm{~mol} \%$ of the glucan and $8.7 \mathrm{~mol} \%$ of the xylan, respectively, in the raw biomass (note: the weight per weight percentages of glucose and xylose were calculated as the dehydrated glucan and xylan, respectively). The formaldehyde-stabilized lignin will be isolated as a light-brown powder (Step 23), representing 21.4\% (wt/wt) (1.0846 g) of the raw, unextracted biomass after correcting for the formaldehyde stabilization. Hydrogenolysis of this powder (Steps 117-128) will yield 33\% (wt/wt) as monomers (after correction for hydrodeoxygenation), for an overall yield of monomers of $7.78 \%$ (wt/wt) versus dry biomass (7.26\% (wt/wt) versus the raw, unextracted biomass). The diformylxylose will be isolated as a yellow oil (Step 34), representing 13.0\% (wt/wt) (0.8517 g, corrected for the formaldehyde stabilization and converted to xylan) of the raw, unextracted biomass and $78.2 \mathrm{~mol} \%$ of the xylan. For both biomass samples, ${ }^{1} \mathrm{H}-\mathrm{NMR}$ and ${ }^{13} \mathrm{C}-\mathrm{NMR}$ spectra of the diformylxyloses and HSQC spectra of the stabilized lignins are provided in the Supplementary Information. For a more detailed presentation of these data, please see Tables 1-6.

\title{
Propionaldehyde biomass fractionation protocol
}

After completion of this procedure (Steps 35-83), we anticipate the collection of three separate biomass fractions: cellulose-rich solids, propionaldehyde-stabilized lignin, and dipropylxylose. For birch wood, we expect that the cellulose-rich solids will appear as a fluffy, gray, fibrous powder (Step 49), representing 39.1\% (wt/wt) (1.9425 g) of the raw, unextracted biomass. Enzymatic hydrolysis (Steps $84-95$ ) of this cellulose will yield $77.4 \%$ (wt/wt) as glucose and $10.1 \%$ (wt/wt) as xylose, representing $94.1 \mathrm{~mol} \%$ of the glucan and $22.2 \mathrm{~mol} \%$ of the xylan, respectively, in the raw biomass (note: the weight per weight percentages of glucose and xylose were calculated as the dehydrated glucan and xylan, respectively). The propionaldehyde-stabilized lignin will be isolated as a purplishbrown powder (Step 70), representing 18.5\% (wt/wt) (0.9853 g) of the raw, unextracted biomass after correcting for the propionaldehyde stabilization. Hydrogenolysis of this powder (Steps 117-128) will yield 38\% (wt/wt) as monomers (after correction for hydrodeoxygenation), for an overall yield of monomers of $7.97 \%$ (wt/wt) versus dry biomass (7.47\% (wt/wt) versus the raw, unextracted biomass). The dipropylxylose will be isolated as a yellow oil (Step 83), representing 10.7\% (wt/wt) (0.9257 g, corrected for the propionaldehyde stabilization and converted to xylan) of the raw, unextracted biomass and $60.1 \mathrm{~mol} \%$ of the xylan.

For beech wood, we expect that the cellulose-rich solids will appear as a fluffy, gray, fibrous powder (Step 49), representing 37.9\% (wt/wt) (1.8998 g) of the raw, unextracted biomass. Enzymatic hydrolysis (Steps 84-95) of this cellulose will yield $82.1 \%$ (wt/wt) as glucose and 9.3\% (wt/wt) as xylose, representing $93.7 \mathrm{~mol} \%$ of the glucan and $21.2 \mathrm{~mol} \%$ of the xylan, respectively, in the raw biomass (note: the weight per weight percentages of glucose and xylose were calculated as the dehydrated glucan and xylan, respectively). The propionaldehyde-stabilized lignin will be isolated as a purplish-brown powder (Step 70), representing 20.6\% (wt/wt) (1.0976 g) of the raw, unextracted biomass after correcting for the propionaldehyde stabilization. Hydrogenolysis of this powder (Steps 117-128) will yield 32\% (wt/wt) as monomers (after correction for hydrodeoxygenation), for an overall yield of monomers of $7.49 \%$ (wt/wt) versus dry biomass (6.99\% (wt/wt) versus the raw, unextracted biomass). The dipropylxylose will be isolated as a yellow oil (Step 83), representing 10.3\% (wt/wt) (0.9011 g, corrected for the propionaldehyde stabilization and converted to xylan) of the raw, unextracted biomass and $62.0 \mathrm{~mol} \%$ of the xylan. For both biomass samples, ${ }^{1} \mathrm{H}-\mathrm{NMR}$ and ${ }^{13} \mathrm{C}-\mathrm{NMR}$ spectra of the dipropylxyloses and HSQC spectra of the stabilized lignins are provided in the Supplementary Information. For a more detailed presentation of these data, please see Tables 1-6.

\author{
Analytical data \\ Diformylxylose: (3aR,3bS,7aR,8aR)-tetrahydro-7H-[1,3]dioxolo $\left[4^{\prime}, 5^{\prime}: 4,5\right]$ furo[3,2-d] $[1,3]$ dioxine \\ Appearance: white crystalline solid \\ TLC (3:1 (vol/vol) hexanes: ethyl acetate, visualized with $\left.\mathrm{KMnO}_{4}\right), R_{\mathrm{f}}=0.2$. \\ ${ }^{1} \mathrm{H}-\mathrm{NMR}(400 \mathrm{MHz}$, chloroform- $d): \delta 6.01(\mathrm{~d}, J=4.0 \mathrm{~Hz}, 1 \mathrm{H}), 5.01(\mathrm{~d}, J=20.0 \mathrm{~Hz}, 2 \mathrm{H}), 4.91$ \\ $(\mathrm{d}, J=4.0 \mathrm{~Hz}, 1 \mathrm{H}), 4.58(\mathrm{~d}, J=4.0 \mathrm{~Hz}, 1 \mathrm{H}), 4.40(\mathrm{~d}, J=4.0 \mathrm{~Hz}, 1 \mathrm{H}), 4.22(\mathrm{~s}, 1 \mathrm{H}), 4.21(\mathrm{~d}, J=12.0$ \\ $\mathrm{Hz}, 1 \mathrm{H}), 3.91$ (s, 1H), $3.82(\mathrm{dd}, J=2.0,12.0 \mathrm{~Hz}, 1 \mathrm{H})$. \\ ${ }^{13} \mathrm{C}-\mathrm{NMR}(101 \mathrm{MHz}$, chloroform-d): 105.1, 96.7, 91.6, 83.7, 77.7, 74.8, 65.9. \\ HSQC (chloroform- $d$ ): see Supplementary Fig. 6.
}


Mass spectrometry (GC-MS-EI): calculated for $\mathrm{C}_{7} \mathrm{H}_{11} \mathrm{O}_{5}\left(\mathrm{M}-\mathrm{H}^{+}\right)=173.0$; found $=173.0$.

Dipropylxylose: $\quad(2 \mathrm{R}, 3 \mathrm{aR}, 3 \mathrm{bS}, 5 \mathrm{R}, 7 \mathrm{aR}, 8 \mathrm{aR})-2,5$-diethyltetrahydro-7H-[1,3] dioxolo $\left[4^{\prime}, 5^{\prime}: 4,5\right]$ furo [3,2-d][1,3] dioxine

Appearance: white crystalline solid.

TLC (3:1 (vol/vol) hexanes: ethyl acetate, visualized with $\left.\mathrm{KMnO}_{4}\right), R_{\mathrm{f}}=0.54$.

${ }^{1} \mathrm{H}-\mathrm{NMR}(400 \mathrm{MHz}$, chloroform- $d): \delta 5.91(\mathrm{~d}, J=4.0 \mathrm{~Hz}, 1 \mathrm{H}), 4.84(\mathrm{t}, J=4.0 \mathrm{~Hz}, 1 \mathrm{H}), 4.36(\mathrm{~d}$, $J=4.0 \mathrm{~Hz}, 1 \mathrm{H}), 4.33(\mathrm{t}, J=5.3 \mathrm{~Hz}, 1 \mathrm{H}), 4.20(\mathrm{~d}, J=13.2 \mathrm{~Hz}, 1 \mathrm{H}), 4.15(\mathrm{~d}, J=4.0 \mathrm{~Hz}, 1 \mathrm{H}), 3.97-3.92$ $(\mathrm{m}, 1 \mathrm{H}), 3.86(\mathrm{dd}, J=13.2,2.0 \mathrm{~Hz}, 1 \mathrm{H}), 1.63(\mathrm{qd}, J=7.5,4.6 \mathrm{~Hz}, 2 \mathrm{H}), 1.55$ (qdd, $J=7.5,5.3,1.2 \mathrm{~Hz}$, $2 \mathrm{H}), 0.89$ (t, $J=7.5 \mathrm{~Hz}, 3 \mathrm{H}), 0.84(\mathrm{t}, J=7.5 \mathrm{~Hz}, 3 \mathrm{H})$.

${ }^{13} \mathrm{C}-\mathrm{NMR}(101 \mathrm{MHz}$, chloroform- $d): \delta 105.90,105.18,101.17,84.26,78.25,72.39,65.99,27.72,26.86$, $8.10,7.57$.

HSQC (chloroform- $d$ ): see Supplementary Fig. 11.

Mass spectrometry (APPI): calculated for $\mathrm{C}_{11} \mathrm{H}_{19} \mathrm{O}_{5}\left(\mathrm{M}+\mathrm{H}^{+}\right)=231.1227$; found $=231.1226$.

Dipropylxylose: $\quad(2 \mathrm{~S}, 3 \mathrm{aR}, 3 \mathrm{bS}, 5 \mathrm{R}, 7 \mathrm{aR}, 8 \mathrm{aR})-2,5$-diethyltetrahydro- $7 \mathrm{H}-[1,3]$ dioxolo $\left[4^{\prime}, 5^{\prime}: 4,5\right]$ furo $[3,2-d][1,3]$ dioxine

Appearance: white crystalline solid.

TLC (3:1 (vol/vol) hexanes: ethyl acetate, visualized with $\left.\mathrm{KMnO}_{4}\right), R_{\mathrm{f}}=0.51$.

${ }^{1} \mathrm{H}-\mathrm{NMR}(400 \mathrm{MHz}$, chloroform- $d): \delta 5.98(\mathrm{~d}, J=3.6 \mathrm{~Hz}, 1 \mathrm{H}), 5.10(\mathrm{t}, J=4.5 \mathrm{~Hz}, 1 \mathrm{H}), 4.44(\mathrm{~d}$, $J=3.6 \mathrm{~Hz}, 1 \mathrm{H}), 4.35(\mathrm{t}, J=5.2 \mathrm{~Hz}, 1 \mathrm{H}), 4.25-4.17(\mathrm{~m}, 2 \mathrm{H}), 3.88-3.78(\mathrm{~m}, 2 \mathrm{H}), 1.58(\mathrm{qd}, J=7.5$, $4.5 \mathrm{~Hz}, 2 \mathrm{H}), 1.56$ (qd, $J=7.5,5.2 \mathrm{~Hz}, 2 \mathrm{H}), 0.87(\mathrm{t}, J=7.5 \mathrm{~Hz}, 3 \mathrm{H}), 0.86(\mathrm{t}, J=7.5 \mathrm{~Hz}, 3 \mathrm{H})$.

${ }^{13} \mathrm{C}-\mathrm{NMR}(101 \mathrm{MHz}$, chloroform- $d): \delta 107.75,105.35,101.12,84.33,78.33,74.92,66.23,27.82,27.64$, $8.11,7.44$.

HSQC (chloroform- $d$ ): see Supplementary Fig. 14.

Mass spectrometry (APPI): calculated for $\mathrm{C}_{11} \mathrm{H}_{19} \mathrm{O}_{5}\left(\mathrm{M}+\mathrm{H}^{+}\right)=231.1227$; found $=231.1229$.

\section{Reporting Summary}

Further information on research design is available in the Nature Research Reporting Summary linked to this article.

\section{Data availability}

The exemplary data that were produced in support of the described procedures are available from the corresponding author upon reasonable request.

1. BP. BP Statistical Review of World Energy. https://www.bp.com/content/dam/bp/business-sites/en/global/ corporate/pdfs/energy-economics/statistical-review/bp-stats-review-2018-full-report.pdf (2018).

2. Cox, P. M., Betts, R. A., Jones, C. D., Spall, S. A. \& Totterdell, I. J. Acceleration of global warming due to carbon-cycle feedbacks in a coupled climate model. Nature 408, 184-187 (2000).

3. Sabine, C. L. et al. The oceanic sink for anthropogenic $\mathrm{CO}_{2}$. Science 305, 367-371 (2004).

4. Shuai, L. \& Luterbacher, J. Organic solvent effects in biomass conversion reactions. ChemSusChem $\mathbf{9}$, 133-155 (2016).

5. Van den Bosch, S. et al. Catalytic strategies towards lignin-derived chemicals. Top. Curr. Chem. (Cham). 376, 36 (2018).

6. Isikgor, F. H. \& Becer, C. R. Lignocellulosic biomass: a sustainable platform for the production of bio-based chemicals and polymers. Polym. Chem. 6, 4497-4559 (2015).

7. Kaparaju, P., Serrano, M., Thomsen, A. B., Kongjan, P. \& Angelidaki, I. Bioethanol, biohydrogen and biogas production from wheat straw in a biorefinery concept. Bioresour. Technol. 100, 2562-2568 (2009).

8. Banerjee, A., Dick, G. R., Yoshino, T. \& Kanan, M. W. Carbon dioxide utilization via carbonate-promoted C-H carboxylation. Nature 531, 215-219 (2016).

9. Dick, G. R., Frankhouser, A. D., Banerjee, A. \& Kanan, M. W. A scalable carboxylation route to furan-2,5dicarboxylic acid. Green Chem 19, 2966-2972 (2017).

10. Zakzeski, J., Bruijnincx, P. C. A., Jongerius, A. L. \& Weckhuysen, B. M. The catalytic valorization of lignin for the production of renewable chemicals. Chem. Rev. 110, 3552-3599 (2010).

11. Alvira, P., Tomás-Pejó, E., Ballesteros, M. \& Negro, M. J. Pretreatment technologies for an efficient bioethanol production process based on enzymatic hydrolysis: a review. Bioresour. Technol. 101, 4851-4861 (2010).

12. Ragnar, M. et al. Pulp. in Ullmann's Encyclopedia of Industrial Chemistry (ed. Elvers, B. et al.) 1-92 (WileyVCH, Weinheim, Germany, 2014).

13. Luterbacher, J. S., Martin Alonso, D. \& Dumesic, J. A. Targeted chemical upgrading of lignocellulosic biomass to platform molecules. Green Chem. 16, 4816-4838 (2014). 
14. Tadesse, H. \& Luque, R. Advances on biomass pretreatment using ionic liquids: an overview. Energy Environ. Sci. 4, 3913-3929 (2011).

15. Luterbacher, J. S. et al. Nonenzymatic sugar production from biomass using biomass-derived $\gamma$-valerolactone. Science 343, 277-280 (2014).

16. Shuai, L., Questell-Santiago, Y. M. \& Luterbacher, J. S. A mild biomass pretreatment using $\gamma$-valerolactone for concentrated sugar production. Green Chem. 18, 937-943 (2016).

17. Ragauskas, A. J. et al. Lignin valorization: improving lignin processing in the biorefinery. Science 344, 1246843-1246843 (2014).

18. Sturgeon, M. R. et al. A mechanistic investigation of acid-catalyzed cleavage of aryl-ether linkages: Implications for lignin depolymerization in acidic environments. ACS Sustain. Chem. Eng. 2, 472-485 (2014).

19. Van den Bosch, S. et al. Integrating lignin valorization and bio-ethanol production: on the role of $\mathrm{Ni}^{-\mathrm{Al}_{2} \mathrm{O}_{3}}$ catalyst pellets during lignin-first fractionation. Green Chem. 19, 3313-3326 (2017).

20. Xu, C., Arancon, R. A. D., Labidi, J. \& Luque, R. Lignin depolymerisation strategies: towards valuable chemicals and fuels. Chem. Soc. Rev. 43, 7485-7500 (2014).

21. Roberts, V. M. et al. Towards quantitative catalytic lignin depolymerization. Chem. Eur. J. 17, 5939-5948 (2011).

22. Lai, C. et al. Lignin alkylation enhances enzymatic hydrolysis of lignocellulosic biomass. Energy Fuels 31, 12317-12326 (2017).

23. Shuai, L. et al. Formaldehyde stabilization facilitates lignin monomer production during biomass depolymerization. Science 354, 329-333 (2016).

24. Lan, W., Amiri, M. T., Hunston, C. M. \& Luterbacher, J. S. Protection group effects during $\alpha, \gamma$-diol lignin stabilization promote high-selectivity monomer production. Angew. Chem. Int. Ed. 57, 1356-1360 (2018).

25. Sluiter, J. \& Sluiter, A. Summative Mass Closure: Laboratory Analytical Procedure (LAP) Review and Integration. https://www.nrel.gov/docs/gen/fy11/48087.pdf (National Renewable Energy Laboratory, 2011).

26. Galkin, M. V. \& Samec, J. S. M. Selective route to 2-propenyl aryls directly from wood by a tandem Organosolv and palladium-catalysed transfer hydrogenolysis. ChemSusChem 7, 2154-2158 (2014).

27. Yan, N. et al. Selective degradation of wood lignin over noble-metal catalysts in a two-step process. ChemSusChem 1, 626-629 (2008).

28. Parsell, T. et al. A synergistic biorefinery based on catalytic conversion of lignin prior to cellulose starting from lignocellulosic biomass. Green Chem. 17, 1492-1499 (2015).

29. Van den Bosch, S. et al. Reductive lignocellulose fractionation into soluble lignin-derived phenolic monomers and dimers and processable carbohydrate pulps. Energy Environ. Sci. 8, 1748-1763 (2015).

30. Phongpreecha, T. et al. Predicting lignin depolymerization yields from quantifiable properties using fractionated biorefinery lignins. Green Chem. 19, 5131-5143 (2017).

31. Schutyser, W. et al. Chemicals from lignin: an interplay of lignocellulose fractionation, depolymerisation, and upgrading. Chem. Soc. Rev. 47, 852-908 (2018).

32. Van Den Bosch, S. et al. Tuning the lignin oil $\mathrm{OH}-$ content with $\mathrm{Ru}$ and $\mathrm{Pd}$ catalysts during lignin hydrogenolysis on birch wood. Chem. Commun. 51, 13158-13161 (2015).

33. Van Den Bosch, S. et al. Reductive lignocellulose fractionation into soluble lignin-derived phenolic monomers and dimers and processable carbohydrate pulps. Energy Environ. Sci. 8, 1748-1763 (2015).

34. Pepper, J. M. \& Lee, Y. W. Lignin and related compounds. I. A comparative study of catalysts for lignin hydrogenolysis. Can. J. Chem. 47, 723-727 (1969).

35. Chang, H., Cowling, E. B. \& Brown, W. Comparative studies on cellulolytic enzyme lignin and milled wood lignin of sweetgum and spruce. Holzforschung 29, 153-159 (1975).

36. Maekawa, E., Ichizawa, T. \& Koshijima, T. An evaluation of the acid-soluble lignin determination in analyses of lignin by the sulfuric acid method. J. Wood Chem. Technol. 9, 549-567 (1989).

37. Kaar, W. E. \& Brink, D. L. Simplified analysis of acid soluble lignin. J. Wood Chem. Technol. 11, 465-477 (1991).

38. Yuan, G., Qi, C., Wu, W. \& Jiang, H. Recent advances in organic synthesis with $\mathrm{CO}_{2}$ as $\mathrm{C} 1$ synthon. Curr. Opin. Green Sustain. Chem. 3, 22-27 (2017).

\section{Acknowledgements}

This work was supported by the European Research Council (ERC) under the European Union's Horizon 2020 research and innovation program (starting grant: CATACOAT, no. 758653), the Swiss National Science Foundation through grant PYAPP2_154281, and the École Polytechnique Fédérale de Lausanne. This work was also accomplished within the framework of the Swiss Competence Center for Bioenergy Research (SCCER-BIOSWEET). We thank L. Menin and D. Ortiz of the SSMI mass spectrometry facility at EPFL for their assistance. We thank W. Lan for helpful discussions during the preparation of the manuscript, especially for the structural assignments of the lignin NMRs.

\section{Author contributions}

M.T.A. and G.R.D. developed and performed the aldehyde-based fractionations, cellulose hydrolyses, and lignin hydrogenolyses. M.T.A., G.R.D., and Y.M.Q.-S. performed the cellulose compositional analyses. G.R.D. performed the biomass compositional analyses. The project was conceived of by M.T.A., G.R.D., and J.S.L. and supervised by J.S.L. All authors participated in the preparation of the manuscript.

\section{Competing interests}

The authors declare competing interests. J.S.L. is an inventor on a European patent application (EP16165180.7) that was submitted by EPFL and covers methods for producing lignin monomers from biomass during biomass depolymerization. 


\section{Additional information}

Supplementary information is available for this paper at https://doi.org/10.1038/s41596-018-0121-7.

Reprints and permissions information is available at www.nature.com/reprints.

Correspondence and requests for materials should be addressed to J.S.L.

Journal peer review information: Nature Protocols thanks Robert Brown and other (anonymous) reviewer(s) for their contribution to the peer review of this work.

Publisher's note: Springer Nature remains neutral with regard to jurisdictional claims in published maps and institutional affiliations.

Received: 10 August 2018; Accepted: 20 December 2018;

Published online: 18 February 2019

\section{Related links}

Key references using this protocol

Shuai, L. et al. Science. 354, 329-333 (2016): http://science.sciencemag.org/content/354/6310/329

Lan, W. et al. Angew. Chem. Int. Ed. 57, 1356-1360 (2018): https://onlinelibrary.wiley.com/doi/abs/10.1002/a nie.201710838 


\section{Reporting Summary}

Nature Research wishes to improve the reproducibility of the work that we publish. This form provides structure for consistency and transparency in reporting. For further information on Nature Research policies, see Authors \& Referees and the Editorial Policy Checklist.

\section{Statistics}

For all statistical analyses, confirm that the following items are present in the figure legend, table legend, main text, or Methods section.

n/a Confirmed

\ $\square$ The exact sample size $(n)$ for each experimental group/condition, given as a discrete number and unit of measurement

Х $\square$ A statement on whether measurements were taken from distinct samples or whether the same sample was measured repeatedly

$\square$ The statistical test(s) used AND whether they are one- or two-sided

$\triangle$ Only common tests should be described solely by name; describe more complex techniques in the Methods section.

Х $\square$ A description of all covariates tested

Х $\square$ A description of any assumptions or corrections, such as tests of normality and adjustment for multiple comparisons

\ $\square$ A full description of the statistical parameters including central tendency (e.g. means) or other basic estimates (e.g. regression coefficient)

X $\square$ AND variation (e.g. standard deviation) or associated estimates of uncertainty (e.g. confidence intervals)

$\triangle \square$ For null hypothesis testing, the test statistic (e.g. $F, t, r$ ) with confidence intervals, effect sizes, degrees of freedom and $P$ value noted

$\triangle \square$ Give P values as exact values whenever suitable.

Х $\square$ For Bayesian analysis, information on the choice of priors and Markov chain Monte Carlo settings

Х $\square$ For hierarchical and complex designs, identification of the appropriate level for tests and full reporting of outcomes

Х $\square$ Estimates of effect sizes (e.g. Cohen's $d$, Pearson's $r$ ), indicating how they were calculated

Our web collection on statistics for biologists contains articles on many of the points above.

\section{Software and code}

Policy information about availability of computer code

Data collection Provide a description of all commercial, open source and custom code used to collect the data in this study, specifying the version used OR state that no software was used.

Data analysis Microsoft Excel, Adobe illustrator

For manuscripts utilizing custom algorithms or software that are central to the research but not yet described in published literature, software must be made available to editors/reviewers. We strongly encourage code deposition in a community repository (e.g. GitHub). See the Nature Research guidelines for submitting code \& software for further information.

\section{Data}

Policy information about availability of data

All manuscripts must include a data availability statement. This statement should provide the following information, where applicable:

- Accession codes, unique identifiers, or web links for publicly available datasets

- A list of figures that have associated raw data

- A description of any restrictions on data availability

\section{Field-specific reporting}

Please select the one below that is the best fit for your research. If you are not sure, read the appropriate sections before making your selection. 


\section{Life sciences study design}

All studies must disclose on these points even when the disclosure is negative.
Sample size
Only single experiments were performed
Data exclusions
No data was excluded
Replication
Experiments were reproducible within \%
Randomization
Does not apply.
Blinding
Does not apply.

\section{Reporting for specific materials, systems and methods}

We require information from authors about some types of materials, experimental systems and methods used in many studies. Here, indicate whether each material, system or method listed is relevant to your study. If you are not sure if a list item applies to your research, read the appropriate section before selecting a response.

\begin{tabular}{|c|c|}
\hline $\mathrm{n} / \mathrm{a}$ & Involved in the study \\
\hline & Antibodies \\
\hline & Eukaryotic cell lines \\
\hline & Palaeontology \\
\hline - & Animals and other organisms \\
\hline 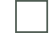 & Human research participants \\
\hline & Clinical data \\
\hline
\end{tabular}

\begin{tabular}{l|l} 
Methods \\
\hline n/a & Involved in the study \\
\hline & $\square$ ChIP-seq \\
$\square$ & $\square$ Flow cytometry \\
\hline & $\square$ MRI-based neuroimaging
\end{tabular}

Antibodies

Antibodies used

Describe all antibodies used in the study; as applicable, provide supplier name, catalog number, clone name, and lot number.

Validation

Describe the validation of each primary antibody for the species and application, noting any validation statements on the manufacturer's website, relevant citations, antibody profiles in online databases, or data provided in the manuscript.

\section{Eukaryotic cell lines}

Policy information about cell lines

Cell line source(s)

State the source of each cell line used.

Authentication

Describe the authentication procedures for each cell line used OR declare that none of the cell lines used were authenticated.

Mycoplasma contamination

Confirm that all cell lines tested negative for mycoplasma contamination OR describe the results of the testing for

mycoplasma contamination OR declare that the cell lines were not tested for mycoplasma contamination.

Commonly misidentified lines (See ICLAC register)

Name any commonly misidentified cell lines used in the study and provide a rationale for their use.

\section{Palaeontology}

Specimen provenance

Provide provenance information for specimens and describe permits that were obtained for the work (including the name of the issuing authority, the date of issue, and any identifying information).

Specimen deposition

Indicate where the specimens have been deposited to permit free access by other researchers.

Dating methods

If new dates are provided, describe how they were obtained (e.g. collection, storage, sample pretreatment and measurement), where they were obtained (i.e. lab name), the calibration program and the protocol for quality assurance OR state that no new dates are provided.

Tick this box to confirm that the raw and calibrated dates are available in the paper or in Supplementary Information. 


\section{Animals and other organisms}

Policy information about studies involving animals; ARRIVE guidelines recommended for reporting animal research

Laboratory animals

For laboratory animals, report species, strain, sex and age OR state that the study did not involve laboratory animals.

Wild animals

Provide details on animals observed in or captured in the field; report species, sex and age where possible. Describe how animals were caught and transported and what happened to captive animals after the study (if killed, explain why and describe method; if released, say where and when) OR state that the study did not involve wild animals.

Field-collected samples

For laboratory work with field-collected samples, describe all relevant parameters such as housing, maintenance, temperature, photoperiod and end-of-experiment protocol OR state that the study did not involve samples collected from the field.

Ethics oversight

Identify the organization(s) that approved or provided guidance on the study protocol, OR state that no ethical approval or guidance was required and explain why not.

Note that full information on the approval of the study protocol must also be provided in the manuscript.

\section{Human research participants}

Policy information about studies involving human research participants

Population characteristics Describe the covariate-relevant population characteristics of the human research participants (e.g. age, gender, genotypic information, past and current diagnosis and treatment categories). If you filled out the behavioural \& social sciences study design questions and have nothing to add here, write "See above."

Recruitment Describe how participants were recruited. Outline any potential self-selection bias or other biases that may be present and how these are likely to impact results.

Ethics oversight

Identify the organization(s) that approved the study protocol.

Note that full information on the approval of the study protocol must also be provided in the manuscript.

\section{Clinical data}

Policy information about clinical studies

All manuscripts should comply with the ICMJE guidelines for publication of clinical research and a completed CONSORT checklist must be included with all submissions.

Clinical trial registration

Provide the trial registration number from ClinicalTrials. gov or an equivalent agency.

Study protocol

Note where the full trial protocol can be accessed OR if not available, explain why.

Data collection

Describe the settings and locales of data collection, noting the time periods of recruitment and data collection.

Outcomes

Describe how you pre-defined primary and secondary outcome measures and how you assessed these measures.

\section{ChIP-seq}

\section{Data deposition}

Confirm that both raw and final processed data have been deposited in a public database such as GEO.

Confirm that you have deposited or provided access to graph files (e.g. BED files) for the called peaks.

Data access links

May remain private before publication.

Files in database submission

Genome browser session (e.g. $\underline{\text { UCSC }})$

\section{Methodology}

Replicates

Sequencing depth

Antibodies
For "Initial submission" or "Revised version" documents, provide reviewer access links. For your "Final submission" document, provide a link to the deposited data.

Provide a list of all files available in the database submission.

Provide a link to an anonymized genome browser session for "Initial submission" and "Revised version" documents only, to enable peer review. Write "no longer applicable" for "Final submission" documents.

Describe the sequencing depth for each experiment, providing the total number of reads, uniquely mapped reads, length of reads and whether they were paired-or single-end.

Describe the antibodies used for the ChIP-seq experiments; as applicable, provide supplier name, catalog number, clone 
Antibodies

Peak calling parameters

Data quality

Software
Specify the command line program and parameters used for read mapping and peak calling, including the ChIP control and index files used.

Describe the methods used to ensure data quality in full detail, including how many peaks are at FDR 5\% and above 5-fold enrichment.

Describe the software used to collect and analyze the ChIP-sea data. For custom code that has been deposited into a community repository, provide accession details.

\section{Flow Cytometry}

Plots

Confirm that:

$\square$ The axis labels state the marker and fluorochrome used (e.g. CD4-FITC).

$\square$ The axis scales are clearly visible. Include numbers along axes only for bottom left plot of group (a 'group' is an analysis of identical markers).

$\square$ All plots are contour plots with outliers or pseudocolor plots.

$\square$ A numerical value for number of cells or percentage (with statistics) is provided.

\section{Methodology}

Sample preparation

Describe the sample preparation, detailing the biological source of the cells and any tissue processing steps used.

Instrument

Identify the instrument used for data collection, specifying make and model number.

Software

Describe the software used to collect and analyze the flow cytometry data. For custom code that has been deposited into a community repository, provide accession details.

Cell population abundance

Describe the abundance of the relevant cell populations within post-sort fractions, providing details on the purity of the samples and how it was determined.

Gating strategy

Describe the gating strategy used for all relevant experiments, specifying the preliminary FSC/SSC gates of the starting cell population, indicating where boundaries between "positive" and "negative" staining cell populations are defined.

Tick this box to confirm that a figure exemplifying the gating strategy is provided in the Supplementary Information.

\section{Magnetic resonance imaging}

\section{Experimental design}

\section{Design type}

Design specifications

Behavioral performance measures

Acquisition

Imaging type(s)

Field strength

Sequence \& imaging parameters

Area of acquisition

Diffusion MRI

Used

\section{Preprocessing}

Preprocessing software
Indicate task or resting state; event-related or block design.

Specify the number of blocks, trials or experimental units per session and/or subject, and specify the length of each tria or block (if trials are blocked) and interval between trials.

State number and/or type of variables recorded (e.g. correct button press, response time) and what statistics were used to establish that the subjects were performing the task as expected (e.g. mean, range, and/or standard deviation across subjects).
Specify: functional, structural, diffusion, perfusion.

Specify in Tesla

Specify the pulse sequence type (gradient echo, spin echo, etc.), imaging type (EPI, spiral, etc.), field of view, matrix size, slice thickness, orientation and TE/TR/flip angle.

State whether a whole brain scan was used OR define the area of acquisition, describing how the region was determined. Not used 
Normalization

Normalization template

Noise and artifact removal

Volume censoring
If data were normalized/standardized, describe the approach(es): specify linear or non-linear and define image types used for transformation OR indicate that data were not normalized and explain rationale for lack of normalization.

Describe the template used for normalization/transformation, specifying subject space or group standardized space (e.g. original Talairach, MNI305, ICBM152) OR indicate that the data were not normalized.

Describe your procedure(s) for artifact and structured noise removal, specifying motion parameters, tissue signals and physiological signals (heart rate, respiration).

Define your software and/or method and criteria for volume censoring, and state the extent of such censoring.

\section{Statistical modeling \& inference}

Model type and settings

Effect(s) tested

Specify type of analysis:

Statistic type for inference

(See Eklund et al. 2016)

\section{Correction}

Models \& analysis

\begin{tabular}{l|l|l|l|l|l}
$\square$ & Functional and/or effective connectivity
\end{tabular} (Carlo).
Specify type (mass univariate, multivariate, RSA, predictive, etc.) and describe essential details of the model at the first and second levels (e.g. fixed, random or mixed effects; drift or auto-correlation).

Define precise effect in terms of the task or stimulus conditions instead of psychological concepts and indicate whether ANOVA or factorial designs were used.

Whole brain $\square$ ROI-based $\square$ Both

Specify voxel-wise or cluster-wise and report all relevant parameters for cluster-wise methods.

Describe the type of correction and how it is obtained for multiple comparisons (e.g. FWE, FDR, permutation or Monte 